\title{
Topological aspects of continuity via generalized limit
}

\author{
Aspectos topológicos de continuidade via limite generalizado
}

\author{
Marcelo Gonçalves Oliveira Vieira \\ Universidade Federal de Uberlândia \\ mgov@ufu.br \\ ORCID:0000-0002-0442-0921
}

\begin{abstract}
This article aims to introduce the concept of generalized continuity of a function with respect to another function and analyse the topological aspects of this concept. Initially, the article presents the concept and the properties of generalized limit of a function with respect to another function, highlighting that the Riemann integral is a particular case of generalized limit. Then, the definition of generalized continuity is presented emphasizing that standard continuity is a particular case of it and hence they do not coincide, in general. Finally, some topological aspects associated with the concept of generalized continuity are approached in order to present proofs about the preservation of topological invariants via generalized continuity, such as preservation of compactness and connectedness.
\end{abstract}

Keywords. Limit. Continuity. Topological invariant.

Mathematics Subject Classification (MSC): primary 54C08; secondary 54C10, 54D05, 54D30, 93C30.

Resumo. Este artigo visa introduzir o conceito de continuidade generalizada de uma função com respeito a outra função e analisar os aspectos topológicos provenientes deste conceito. Inicialmente, o artigo apresenta o conceito e as propriedades de limite generalizado de uma aplicação com respeito a outra aplicação, destacando que integração de Riemann trata-se de um caso particular de limite generalizado. Em seguida, a definição de continuidade generalizada é apresentada enfatizando que a continuidade padrão é um caso particular dela e, portanto, elas não coincidem, em geral. Por fim, alguns aspectos topológicos associados ao 
conceito de continuidade generalizada são abordados a fim apresentar provas sobre a preservação de invariantes topológicos via continuidade generalizada, tais como preservação de compacidade e conexidade.

Palavras-chave. Limite. Continuidade. Invariante topológico.

\section{Introduction}

In [10] the authors considered Young systems and their trajectories, which are generated by Young integrals strongly dependent on integrators. These integrators have a role similar to the control parameters that generate trajectories of control systems as in [3]. Nonetheless, trajectories, integrated functions and integrators for Young systems may fail to have differentiability property. However, to study specific trajectories of the Young systems in [11], called regular trajectories, it is important to have results similar to those presented in control systems with some differentiability, such as formulas about dependence on parameters. Some of these results present in [11] have analogues in systems of differential equations, but the proofs of these results cannot make use of techniques that involve differentiability, precisely due to the lack of this characteristic in Young systems. Therefore, in [11] Vieira, Kizil and Catuogno focused on the fact that integrals and derivatives are special cases of limits and hence when they operated at the level of definition of limits, they obtained proofs of the expected results in the context of the Young systems.

Considering the possibility that the notion of limit may contain unrevealed information, it is convenient to ask if limits admit some type of control parameter. In fact, it was evidenced in [2] that usual limits are driven by identity functions and it was proposed that this notion can be expanded admitting that limits can be directed by other types of functions. In summary, it was presented in [2] a notion of limit named by generalized limit. The notion of generalized limit leads naturally to a concept of generalized continuity and this can be seen briefly, but without due rigor, in [12].

This article aims to formalize the concept of generalized continuity inspired by the notion of generalized limit, moreover, it has the intention of work some topological implications arising from the concept of generalized continuity.

The terminology "generalized continuity" has already been addressed in other contexts and with other meanings, for example, in the recent works proposed by Kupka in [5], [6], [7] and [8]. The concept of generalized continuity present in this article does not coincide with the notion of continuity proposed by Kupka and this is evidenced in the article through examples. 
This article is organized to bring a general and thorough view to the reader. In this introduction, results about usual notions of limit and continuity are reviewed so that a parallel can be made between this usual notions and the proposed generalized notions. Subsequently, the reader has a brief explanation of the motivation that led to formulation of the generalized limit concept. Next, the reader is presented to the notion of generalized continuity in the context of metric spaces. The next step is to approach the generalized continuity in the context of topological spaces and in this part are located the definitions, theorems and examples most robust of the article. The following section is dedicated to highlighting the differences between generalized continuity via generalized limit and continuity in the sense proposed by Kupka. Finally, there is a section to deal with some topological invariants (compactness and connectedness) in the light of generalized continuity.

This article will assume that the reader is familiar with notions about topology of metric spaces and general topology. Thus, in this introduction the statements of the next propositions are mentioned, however their proofs are omitted. Further details about the propositions mentioned can be seen in [4] and [9].

Although the general properties about image and preimage of functions are elementary results, these properties are used recurrently and crucially in the proofs of propositions and theorems proposed in the next sections of this article. For this reason these properties are emphasized here.

Proposition 1. Let $X$ and $Y$ be sets and let $f: X \rightarrow Y$ be a function. The following statements hold true:

(i) $f(A)=\emptyset$ if, and only if, $A=\emptyset$.

(ii) If $A_{1} \subset A_{2} \subset X$, then $f\left(A_{1}\right) \subset f\left(A_{2}\right)$.

(iii) If $A_{1}, A_{2} \subset X$, then $f\left(A_{1} \cup A_{2}\right)=f\left(A_{1}\right) \cup f\left(A_{2}\right)$.

(iv) If $A_{1}, A_{2} \subset X$, then $f\left(A_{1} \cap A_{2}\right) \subset f\left(A_{1}\right) \cap f\left(A_{2}\right)$.

Proposition 2. Let $X$ and $Y$ be sets and let $f: X \rightarrow Y$ be a function. The following statements hold true:

(i) If $B_{1} \subset B_{2} \subset Y$, then $f^{-1}\left(B_{1}\right) \subset f^{-1}\left(B_{2}\right)$.

(ii) If $B_{1}, B_{2} \subset Y$, then $f^{-1}\left(B_{1} \cup B_{2}\right)=f^{-1}\left(B_{1}\right) \cup f^{-1}\left(B_{2}\right)$.

(iii) If $B_{1}, B_{2} \subset Y$, then $f^{-1}\left(B_{1} \cap B_{2}\right)=f^{-1}\left(B_{1}\right) \cap f^{-1}\left(B_{2}\right)$.

It is also important to highlight results that merge image and preimage of a function. 
Proposition 3. Let $X$ and $Y$ be sets and let $f: X \rightarrow Y$ be a function. The following statements hold true:

(i) If $A \subset X$, then $A \subset f^{-1}(f(A))$.

(ii) If $A \subset X$ and $f$ is injective, then $A=f^{-1}(f(A))$.

(iii) $f^{-1}(f(X))=X$.

(iv) If $B \subset Y$, then $f\left(f^{-1}(B)\right) \subset B$.

(v) If $B \subset Y$ and $f$ is surjective, then $f\left(f^{-1}(B)\right)=B$.

Proposition 4. Let $X$ and $Y$ be sets and let $f: X \rightarrow Y$ be a function. The following statements hold true:

(i) If $A \subset X$ and $B \subset Y$, then $B \cap f(A)=f\left(f^{-1}(B) \cap A\right)$.

(ii) If $B \subset f(A)$, then there exists $C \subset A$ such that $f(C)=B$.

(iii) $f(A) \subset B$ if, and only if, $A \subset f^{-1}(B)$.

Definition 1. Let $\mathbb{R}$ be equipped with the usual metric, let $f: X \subset \mathbb{R} \rightarrow \mathbb{R}$ be a function and let a be an accumulation point in $X$. A real number $b$ is said to be a limit of $f(x)$ as $x$ approaches a if, for every $\epsilon>0$, there exists a $\delta>0$ such that

$$
x \in X \text { and } 0<|x-a|<\delta \text { implies }|f(x)-b|<\epsilon .
$$

Since the codomain of the function in the previous definition is $\mathbb{R}$ and since $\mathbb{R}$ is a Hausdorff space, being $b$ a limit of $f(x)$ as $x$ approaches $a$, then $b$ is unique, in the sense of to satisfy condition (1) and in this case $b$ is denoted, without any doubt, by

$$
b=\lim _{x \rightarrow a} f(x) .
$$

In other words, the above definition means $f(x)$ is arbitrarily close to $b$, as long as $x \in X$ is sufficiently close to $a$.

In the definition of a limit, it is required that $a$ is an accumulation point in $X$, because this guarantees the existence of points in the domain $X$ of the function $f$ that are close to $a$. However, it is not necessary for $a$ to belong to $X$, that is, it is not necessary that $f$ is defined at the point $a$ for the limit of $f(x)$ to exist as $x$ approaches $a$.

In mathematical literature there are works in which the condition (1) of Definition 1 is replaced by the following condition:

$$
x \in X \text { and }|x-a|<\delta \text { implies }|f(x)-b|<\epsilon .
$$


In this case, a real number $b$ that satisfies condition (2) is said to be a non-deleted limit of $f(x)$ as $\mathrm{x}$ approaches $a$. Considering the context in which $a$ is an accumulation point in $\mathrm{X}$, the notion of non-deleted limit is more restrictive than the notion of limit (deleted limit) given by Definition 1, because there are cases of functions that admit limit, but do not admit non-deleted limit. For example, considering $f: \mathbb{R} \rightarrow \mathbb{R}$ given by

$$
f(x)=\left\{\begin{array}{l}
2, \text { se } x=0 \\
1, \text { se } x \neq 0
\end{array},\right.
$$

then $\lim _{x \rightarrow 0} f(x)=1$, but there is not non-deleted limit of $f(x)$ as $x$ approaches 0 , because for every real number $b$, taking $\epsilon=\frac{1}{2}$, the condition (2) fails, for all $\delta>0$.

It is important to note that in most mathematical literature the Definition 11 is not considered for cases in which $a$ is an isolated point of $X$, because in this situation, every real number $b$ becomes a limit of $f(x)$ as $x$ approaches $a$. In fact, if $a$ is an isolated point of $X$, then there is $r>0$ such that the open ball with center $a$ and radius $r$ is the unit set $\{a\}$. Suppose that a real number $b$ does not satisfy condition (1). In this case, there is $\epsilon>0$ such that for every $\delta>0$, there exists $x_{\delta} \in\{x \in X: 0<|x-a|<\delta\}$ satisfying $\left|f\left(x_{\delta}\right)-b\right| \geq \epsilon$. In particular, for $\delta=r$, there exists $x_{r} \in\{x \in X: 0<|x-a|<r\}$ and $\left|f\left(x_{r}\right)-b\right| \geq \epsilon$, but this is a contradiction, because the set $\{x \in X: 0<|x-a|<r\}$ is empty. Therefore, if $a$ is an isolated point of $X$, every real number $b$ satisfies condition (1). Now, if $a$ is an isolated point of $X$, only the number $f(a)$ satisfies condition (2).

The definition of usual limit for a function can be reproduced for functions between metric spaces.

Definition 2. Let $\left(M, d_{M}\right)$ and $\left(N, d_{N}\right)$ be metric spaces, let $X$ be a subset of $M$, let $f: X \subset\left(M, d_{M}\right) \rightarrow\left(N, d_{N}\right)$ be a function and let a be an accumulation point in $X . A$ point $b \in N$ is said to be a limit of $f(x)$ as $x$ approaches a if, for every $\epsilon>0$, there exists a $\delta>0$ such that

$$
x \in X \text { and } 0<d_{M}(x, a)<\delta \text { implies } d_{N}(f(x), b)<\epsilon .
$$

Given a metric space $\left(M, d_{M}\right)$ and given $X$ a subset $M$, we can consider $X$ as metric subspace of $\left(M, d_{M}\right)$. Thus, without loss of generality, we can simply consider the domains of functions as metric spaces.

Definition 3. Let $\left(M, d_{M}\right)$ and $\left(N, d_{N}\right)$ be metric spaces and let $a \in M$. A function $f:\left(M, d_{M}\right) \rightarrow\left(N, d_{N}\right)$ is said to be continuous at a if, for every $\epsilon>0$, there exists a 
$\delta>0$ such that

$$
x \in M \text { e } d_{M}(x, a)<\delta \text { implies } d_{N}(f(x), f(a))<\epsilon .
$$

A function $f:\left(M, d_{M}\right) \rightarrow\left(N, d_{N}\right)$ is said to be continuous if $f$ is continuous at $a$, for all $a \in M$.

Proposition 5. Let $\left(M, d_{M}\right)$ and $\left(N, d_{N}\right)$ be metric spaces and let $a \in M$. A function $f:\left(M, d_{M}\right) \rightarrow\left(N, d_{N}\right)$ is continuous at a if, and only if, a is isolated point of $M$ or

$$
\lim _{x \rightarrow a} f(x)=f(a)
$$

The definition of continuity for a function between metric spaces can be rewritten, in an equivalent way, using the language of open balls in metric spaces.

Proposition 6. Let $\left(M, d_{M}\right)$ and $\left(N, d_{N}\right)$ be metric spaces and let a $\in M$. A function $f:\left(M, d_{M}\right) \rightarrow\left(N, d_{N}\right)$ is said be continuous at a if, and only if, for every $\epsilon>0$, there exists a $\delta>0$ such that

$$
f\left(B_{M}(a ; \delta)\right) \subset B_{N}(f(a) ; \epsilon)
$$

Proposition 7. Let $\left(M, d_{M}\right)$ and $\left(N, d_{N}\right)$ be metric spaces. We have that a function $f:\left(M, d_{M}\right) \rightarrow\left(N, d_{N}\right)$ is continuous if, and only if, for every open subset $U$ of $N$ (in metric sense), it is valid that $f^{-1}(U)$ is an open subset of $M$ (in metric sense).

In view of the previous proposition, in general topology the definition of continuity is expanded to the context of the functions between topological spaces, as can be seen in the next definition. Before, a notation is introduced to simplify the dictations about open sets with respect to a topology. Let $(X, \mathcal{T})$ be a topological space and let $p \in X$, then the collection of all open sets containing $p$, with respect the topology $\mathcal{T}$, is denoted by $\mathcal{T}_{p}$.

Definition 4. Let $\left(X, \mathcal{T}^{X}\right)$ and $\left(Y, \mathcal{T}^{Y}\right)$ be topological spaces, let $f:\left(X, \mathcal{T}^{X}\right) \rightarrow\left(Y, \mathcal{T}^{Y}\right)$ be a function and let $a \in X$. We say that $f$ is continuous at $a$ iffor every $U \in \mathcal{T}_{f(a)}^{Y}$, there exists a $V \in \mathcal{T}_{a}^{X}$ such that

$$
f(V) \subset U \text {. }
$$

We simply say that a function $f:\left(X, \mathcal{T}^{X}\right) \rightarrow\left(Y, \mathcal{T}^{Y}\right)$ is continuous if $f$ is continuous at $a$, for all $a \in X$.

Proposition 8. Let $\left(X, \mathcal{T}^{X}\right)$ and $\left(Y, \mathcal{T}^{Y}\right)$ be topological spaces. We have that a function $f:\left(X, \mathcal{T}^{X}\right) \rightarrow\left(Y, \mathcal{T}^{Y}\right)$ is continuous if, and only if, $f^{-1}(U) \in \mathcal{T}^{X}$, for all $U \in \mathcal{T}^{Y}$. 


\section{Generalized limit}

When observing the definition of the Riemann integral, it is verified that the limit used in its definition is not precisely the usual limit treated in courses of Differential and Integral Calculus and Real Analysis, because the sum used in this definition depends on a partition, while the limit applied to the sum is not calculated with respect to the partition, but calculated with respect to the norm of the partition, when it tends to zero.

Considering this observation, Vieira and Braz proposed in [2] a generalization of this special situation observed for limits of the Riemann sums and they called the limits of functions proposed in this generalization by generalized limits. It was verified that the definition of generalized limit allows one to prove properties and results similar to those existing for the usual limits.

First, let us remember the definition of Riemann integral of a bounded real function defined on a compact interval. For this we need to present some previous arguments about partitions of intervals.

A partition of the interval $[a, b]$ is a finite set $P=\left\{t_{0}<t_{1}<\ldots<t_{k}\right\}$, with $t_{0}=a$ and $t_{k}=b$. Each interval $\left[t_{i-1}, t_{i}\right]$ with length $t_{i}-t_{i-1}$ is named $\mathrm{o} i$-th interval of partition $P$. The set of all partitions of the $[a, b]$ is denoted by $\mathrm{P}([a, b])$. The norm of a partition $P=\left\{t_{0}<t_{1}<\ldots<t_{n}\right\} \subset[a, b]$ is the number $\|P\|=\max \left\{\left(t_{i}-t_{i-1}\right): t_{i} \in P\right\}$. Moreover, a tagged partition of the interval $[a, b]$ is a pair $P^{*}=(P, \varepsilon)$, where $P$ is a partition of the interval $[a, b]$ and $\varepsilon=\left(\varepsilon_{1}, \ldots, \varepsilon_{n}\right)$ is a list with $n$ numbers such that $t_{i-1} \leq \varepsilon_{i} \leq t_{i}$, for each $i=1,2, \ldots, n$. The norm of a dotted partition $P^{*}=(P, \varepsilon)$ of the interval $[a, b]$ is defined as the value of the norm of $P$, that is, $\left\|P^{*}\right\|=\|P\|$. The set of all partitions of the interval $[a, b]$ is denoted by $\mathrm{P}^{*}([a, b])$.

Definition 5. Let $f:[a, b] \rightarrow \mathbb{R}$ be a bounded function and let $P^{*}=(P, \varepsilon)$ be a tagged partition of interval $[a, b]$, with $P=\left\{t_{0}<t_{1}<\ldots<t_{n}\right\}$ and $\varepsilon=\left(\varepsilon_{1}, \ldots, \varepsilon_{n}\right)$. The Riemann sum of $f$ with respect to $P^{*}$ is the number $\mathcal{R}_{f}\left(P^{*}\right)$ given by

$$
\mathcal{R}_{f}\left(P^{*}\right)=\sum_{i=1}^{n} f\left(\varepsilon_{i}\right) \cdot\left(t_{i}-t_{i-1}\right)=\sum_{\left(t_{i}, \varepsilon_{i}\right) \in P^{*}} f\left(\varepsilon_{i}\right) \cdot\left(t_{i}-t_{i-1}\right) .
$$

Definition 6. Let $f:[a, b] \rightarrow \mathbb{R}$ be a function. The Riemann sum $\mathcal{R}_{f}\left(P^{*}\right)$ tends to $b \in \mathbb{R}$ as $\left\|P^{*}\right\|$ approaches 0 , and is written

$$
b=\lim _{\left\|P^{*}\right\| \rightarrow 0}\left(\sum_{\left(t_{i}, \varepsilon_{i}\right) \in P^{*}} f\left(\varepsilon_{i}\right) \cdot\left(t_{i}-t_{i-1}\right)\right)
$$

Braz. Elect. J. Math., Ituiutaba, v.2 - n.3, jan/jun 2021, p. 70-107. 
iffor every $\epsilon>0$, there exists $a \delta>0$ such that $\left|\mathcal{R}_{f}\left(P^{*}\right)-b\right|<\epsilon$, for all $P^{*} \in \mathrm{P}^{*}([a, b])$, with $\left\|P^{*}\right\|<\delta$. The number $b$ is called Riemann integral of $f$ in the interval $[a, b]$ and is denoted by $\int_{a}^{b} f(x) d x$.

Note that the idea of limit present in the definition of Riemann integral is not exactly a usual limit, since for a usual limit of function is required of objects that tend to a point of accumulation $a$ to be points of the domain of this function. In a Riemann integral the mathematical objects that tend to 0 are the norms of the tagged partitions and not the partitions themselves, while the Riemann sum is described as a function of tagged partitions and not a function of norms of these partitions.

Also note that in the usual limit $\lim _{x \rightarrow a} f(x)$ the points $x$ that tend to $a$ can be rewritten as $x=i d(x)$, where $i d$ denotes the identity function defined in $X$, which is the domain of the function $f$. Thus, the limit can be written as

$$
\lim _{x \rightarrow a} f(x)=\lim _{i d(x) \rightarrow a} f(x) .
$$

A natural question arises: can we exchange $i d(x)$ with a function $n$ (which has the same domain of the function $f$ ) in the notation of the usual limit of $f$ and this notation to continue with some pertinent mathematical sense?

The question before and the observation made about the limit present in Riemann integral are motivators for the definition of generalized limit of a function, which is a good generalization for the definition of the usual limit, in the sense that it is possible to obtain properties and results similar to those already known about the usual limits.

Definition 7. Let $X$ be a non-empty set, let $\left(Y, d_{Y}\right)$ and $\left(Z, d_{Z}\right)$ be metric spaces, let $f: X \rightarrow\left(Y, d_{Y}\right)$ and $n: X \rightarrow\left(Z, d_{Z}\right)$ be functions and let $z$ be an accumulation point in the image $n(X)$. We say that the point $y \in Y$ is generalized limit of $f(x)$ as $n(x)$ approaches $z$ iffor every $\epsilon>0$, there exists $a \delta>0$ such that

$$
x \in X \text { and } 0<d_{Z}(n(x), z)<\delta \text { implies } d_{Y}(f(x), y)<\epsilon .
$$

Proposition 9. Let $X$ be a non-empty set, let $\left(Y, d_{Y}\right)$ and $\left(Z, d_{Z}\right)$ be metric spaces, let $f: X \rightarrow\left(Y, d_{Y}\right)$ and $n: X \rightarrow\left(Z, d_{Z}\right)$ be functions and let $z$ be an accumulation point in $n(X)$. If $y$ is generalized limit of $f(x)$ as $n(x)$ approaches $z$ e $y^{\prime}$ is generalized limit of $f(x)$ as $n(x)$ approaches $z$, then $y=y^{\prime}$.

Demonstraçãa. Suppose $y \neq y^{\prime}$. We have that $d_{Y}\left(y, y^{\prime}\right)>0$ and $\epsilon=\frac{d_{Y}\left(y, y^{\prime}\right)}{2}>0$. Since $y$ is generalized limit of $f(x)$ as $n(x)$ approaches $z$, it follows that for the $\epsilon$ considered, 
there exists a $\delta_{1}>0$ such that

$$
x \in X \text { and } 0<d_{Z}(n(x), z)<\delta_{1} \text { implies } d_{Y}(f(x), y)<\epsilon .
$$

Similarly, since $y^{\prime}$ is generalized limit of $f(x)$ as $n(x)$ approaches $z$, it follows that for the $\epsilon$ considered, there exists a $\delta_{2}>0$ such that

$$
x \in X \text { and } 0<d_{Z}(n(x), z)<\delta_{2} \text { implies } d_{Y}\left(f(x), y^{\prime}\right)<\epsilon .
$$

Taking $\delta=\min \left\{\delta_{1}, \delta_{2}\right\}$ and using the hypothesis that $z$ is point of accumulation in $n(X)$, then there exists $x_{0} \in X$ such that $n\left(x_{0}\right) \in B_{Z}(z, \delta)-\{z\}$, that is, $0<d_{Z}\left(n\left(x_{0}\right), z\right)<\delta$. It follows from expressions (5) and (6) that

$$
d_{Y}\left(y, y^{\prime}\right) \leq d_{Y}\left(y, f\left(x_{0}\right)\right)+d_{Y}\left(f\left(x_{0}\right), y^{\prime}\right)<\epsilon+\epsilon=d_{Y}\left(y, y^{\prime}\right),
$$

which is a contradiction. Therefore, $y=y^{\prime}$.

Once one has uniqueness for the generalized limit of a function with respect to another, as seen in Proposition 9, one can use an unequivocal notation to represent generalized limits. The generalized limit of a function $f$ with respect to a function $n$ and a point $z$, if this exists, is denoted by

$$
\underset{n(x) \rightarrow z}{\operatorname{glim}} f(x)
$$

Example 1. The most obvious example of a generalized limit is the Riemann integral of an integrable function $f:[a, b] \rightarrow \mathbb{R}$. In fact, considering $\mathbb{R}$ equipped with the usual metric, considering the function $\mathcal{R}_{f}: \mathrm{P}^{*}([a, b]) \rightarrow \mathbb{R}$ that associates each tagged partition $P^{*}$ of $[a, b]$ to the Riemann sum of $f$ with respect to $P^{*}$ and considering the function $\mathcal{N}: \mathrm{P}^{*}([a, b]) \rightarrow \mathbb{R}$ that associates each tagged partition $P^{*}$ of $[a, b]$ to its norm, we have by the definition of Riemann integral of $f$ that this is the generalized limit of the function $\mathcal{R}_{f}$ with respect to the function $\mathcal{N}$ and the point $0 \in \mathbb{R}$, this is,

$$
\int_{a}^{b} f(x) d x=\underset{\mathcal{N}\left(P^{*}\right) \rightarrow 0}{\operatorname{glim}} \mathcal{R}_{f}\left(P^{*}\right)
$$

Example 2. Consider $f: \mathbb{R} \rightarrow \mathbb{R}$ given by

$$
f(x)=\left\{\begin{array}{l}
x, \text { if } x<1 \\
3, \text { if } x=1 \\
x+1, \text { if } x>1
\end{array}\right.
$$


and $n: \mathbb{R} \rightarrow \mathbb{R}$ given by

$$
n(x)=\left\{\begin{array}{l}
0, \text { if } x=1 \\
x-2, \text { if } x \neq 1
\end{array}\right.
$$

Note that the usual limit $\lim _{x \rightarrow 1} f(x)$ does not exist.

However, we have that $\underset{n(x) \rightarrow n(1)}{\operatorname{glim}} f(x)=\underset{n(x) \rightarrow 0}{\operatorname{glim}} f(x)=3$. In fact, given $\epsilon>0$, take $\delta=$ $\min \{1, \epsilon\}$. Hence, if $0<|n(x)-0|<\delta$, this results in $0<|x-2|=|(x-2)-0|<\delta \leq 1$, which implies $x \in(1,2) \cup(2,3)$ and therefore $|f(x)-3|=|(x+1)-3|=|x-2|<\delta \leq \epsilon$.

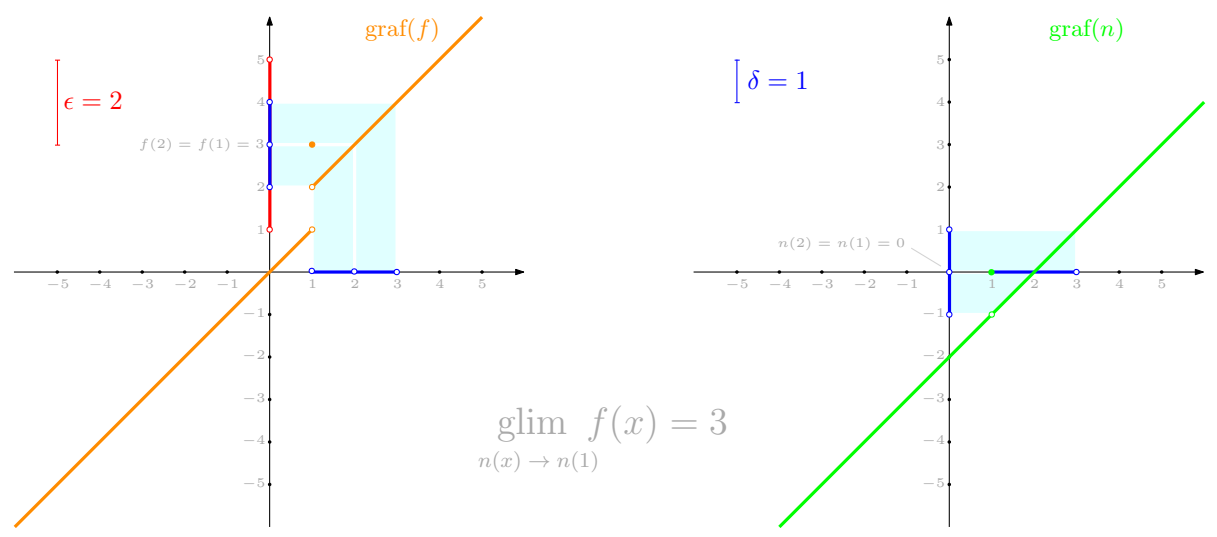

Figure 1: an example of generalized limit

With this last example, we see that the generalized limit and usual limit are not equivalent concepts. Every usual limit is a generalized limit with respect to the identity function, however the existence of a generalized limit for a function $f$ with respect to some other function does not imply the existence of the usual limit for $f$.

Another important point to highlight is that in the definition of generalized limit of a function $f$ with respect to a function $n$, it is not necessary to require any type of mathematical structure (topology, metric, norm, internal product, vector operations, etc.) in the domains of $f$ and $n$, it is necessary metric structure only in the codomains of these functions. Note that in the first example, in the domain $\mathrm{P}^{*}([a, b])$ of the functions $\mathcal{R}_{f}$ and $\mathcal{N}$ it was not considered any structure.

Next, we list some results about generalized limits and their proofs can be seen in [2]. These results allow the reader to realize that the concept of generalized limit has similar properties to those already known in the usual limits.

Proposition 10. Let $X$ be a non-empty set, let $\left(Y, d_{Y}\right)$ and $\left(Z, d_{Z}\right)$ be metric spaces, let 
$f: X \rightarrow\left(Y, d_{Y}\right)$ and $n: X \rightarrow\left(Z, d_{Z}\right)$ be functions and let $z$ an accumulation point in $n(X)$. Then $\underset{n(x) \rightarrow z}{\operatorname{glim}} f(x)=y$ if, and only if, for every sequence $\left(x_{k}\right)_{k=1}^{\infty} \subset X$, with $n\left(x_{k}\right) \rightarrow z$, we have $f\left(x_{k}\right) \rightarrow y$.

Proposition 11. Let $X$ be a non-empty set, let $(Y,\|\cdot\|)$ be a $\mathbb{R}$-normed space, let $\left(Z, d_{Z}\right)$ be a metric space and let $f: X \rightarrow(Y,\|\cdot\|), g: X \rightarrow(Y,\|\cdot\|)$ and $n: X \rightarrow\left(Z, d_{Z}\right)$ be functions such that the generalized limits $\underset{n(x) \rightarrow z}{\operatorname{glim}} f(x)=y$ and $\underset{n(x) \rightarrow z}{\operatorname{glim}} g(x)=w$ exist. The following statements hold true:

(i) $\underset{n(x) \rightarrow z}{\operatorname{glim}}(f+g)(x)=y+w=\underset{n(x) \rightarrow z}{\operatorname{glim}} f(x)+\underset{n(x) \rightarrow z}{\operatorname{glim}} g(x)$.

(ii) If $\lambda \in \mathbb{R}$, then $\underset{n(x) \rightarrow z}{\operatorname{glim}} \lambda \cdot f(x)=\lambda \cdot y=\lambda \cdot \underset{n(x) \rightarrow z}{\operatorname{glim}} f(x)$.

Proposition 12. Let $X$ be a non-empty set, let $\mathbb{R}$ be equipped with the usual metric, let $\left(Z, d_{Z}\right)$ be a metric space and let $f: X \rightarrow \mathbb{R}, g: X \rightarrow \mathbb{R}$ and $n: X \rightarrow Z$ be functions such that the generalized limits $\underset{n(x) \rightarrow z}{\operatorname{glim}} f(x)=y$ and $\underset{n(x) \rightarrow z}{\operatorname{glim}} g(x)=w$ exist. The following statements hold true:

(i) $\underset{n(x) \rightarrow z}{\operatorname{glim}}(f \cdot g)(x)=y \cdot w=\underset{n(x) \rightarrow z}{\operatorname{glim}} f(x) \cdot \underset{n(x) \rightarrow z}{\operatorname{glim}} g(x)$.

(ii) $\underset{n(x) \rightarrow z}{\operatorname{glim}} \frac{f(x)}{g(x)}=\frac{y}{w}$, since that $w \neq 0$.

\section{Generalized continuity in metric context}

A question that arises about generalized limits of functions is: Can generalized limits lead to some concept of continuity, in the same way that classical continuity can be characterized through usual limits of functions? The answer to this question is yes, and we present in the next sections of this article the details of the idea of continuity based on generalized limits.

Since the concept of continuity for functions between metric spaces is directly related to the usual limits of these functions with respect to points of their domains, it is possible to propose a generalization of the concept of continuity for functions between metric spaces based on the concept of generalized limits for functions between metric spaces.

Definition 8. Let $X$ be a non-empty set, let $\left(Y, d_{Y}\right)$ and $\left(Z, d_{Z}\right)$ be metric spaces and let $f: X \rightarrow\left(Y, d_{Y}\right)$ and $n: X \rightarrow\left(Z, d_{Z}\right)$ be functions and let $a \in X$. The function $f$ is said 
be continuous with respect to $n$ (or $n$-continuous) at a if for every $\epsilon>0$, there exists a $\delta>0$ such that

$$
x \in X \text { and } d_{Z}(n(x), n(a))<\delta \text { implies } d_{Y}(f(x), f(a))<\epsilon .
$$

Theorem 1. Let $X$ be a non-empty set, let $\left(Y, d_{Y}\right)$ and $\left(Z, d_{Z}\right)$ be metric spaces, let $f: X \rightarrow\left(Y, d_{Y}\right)$ and $n: X \rightarrow\left(Z, d_{Z}\right)$ be functions and let $a \in X$. The following statements hold true:

(i) If $f$ is $n$-continuous at a, then $n(a)$ is isolated point of the image $n(X)$ or

$$
\operatorname{glim}_{n(x) \rightarrow n(a)} f(x)=f(a)
$$

(ii) If $n(a)$ is isolated point of the image $n(X)$ and $n^{-1}(\{n(a)\})=\{a\}$, then $f$ is $n$ continuous at a.

(iii) If $\underset{n(x) \rightarrow n(a)}{\operatorname{glim}} f(x)=f(a)$ and $n^{-1}(\{n(a)\})=\{a\}$, then $f$ is $n$ - continuous at a.

Demonstraçãoo. (i) Let $a \in X$ such that $n(a)$ is not isolated point of $n(X)$. Thus, $n(a)$ is an accumulation point in $n(X)$. Given $\epsilon>0$, since $f$ is $n$-continuous at $a$, then there exists a $\delta>0$ such that the condition (7) is satisfied and, in particular, the condition (4) is satisfied for $z=n(a)$ and $y=f(a)$. Therefore, $\underset{n(x) \rightarrow n(a)}{\operatorname{glim}} f(x)=f(a)$.

(ii) Note that $d_{Z}(n(x), n(a))=0$ implies $n(x)=n(a)$ and this implies $x=a$, because $n^{-1}(\{n(a)\})=\{a\}$ by hypothesis. Since $n(a)$ is an isolated point of $n(X)$, then there exists a $\delta>0$ such that $B_{Z}(n(a) ; \delta) \cap n(X)=\{n(a)\}$. Hence, given $\epsilon>0$ and using this $\delta>0$, then $d_{Z}(n(x), n(a))<\delta$ implies $x=a$ and this implies $d_{Y}(f(x), f(a))=d_{Y}(f(a), f(a))=0<\epsilon$. Therefore, $f$ is continuous at $a$.

(iii) If $\underset{n(x) \rightarrow n(a)}{\operatorname{glim}} f(x)=f(a)$, then given $\epsilon>0$, there exists a $\delta>0$ such that the condition (4) is satisfied for $z=n(a)$ and $y=f(a)$. We have that $d_{Z}(n(a), n(a))=0<\delta$ implies $d_{Y}(f(a), f(a))=0<\epsilon$. It is known that if $n^{-1}(\{n(a)\})=\{a\}$ and $d_{Z}(n(x), n(a))=0$, then $x=a$. Hence, there is no $x \in X$, with $x \neq a$, such that $d_{Z}(n(x), n(a))=0<\delta$ and $d_{Y}(f(x), f(a)) \geq \epsilon$. Thus, given $\epsilon>0$, there exists a $\delta>0$ such that the condition (7) is satisfied. Therefore, $f$ is $n$-continuous at $a$.

It is important to highlight that in Definition 7 about generalized limit the condition (4) disregards all points $x \in X$ such that $n(x)=n(a)$, that is, it considers only points $x \in X$ such that $0<d(n(x), n(a))<\delta$. The condition (4) is established in this way 
for that Definition 7 be a generalization in accordance with the definition of usual limit. However, if condition (4) is modified to consider the points $x \in X$ such that $n(x)=n(a)$ or $d(n(x), n(a)$, generating a more specific concept of generalized limit, then it is easy to see that statements (ii) and (iii) of Theorem 1 remain valid by removing the hypothesis $n^{-1}(\{n(a)\})=\{a\}$. Note that when $n$ is the identity function, it becomes indifferent for this case to consider condition (4) or its modification mentioned.

Example 3. As mentioned earlier, for functions $f: \mathbb{R} \rightarrow \mathbb{R}$ given by

$$
f(x)=\left\{\begin{array}{l}
x, \text { if } x<1 \\
3, \text { if } x=1 \\
x+1, \text { if } x>1
\end{array}\right.
$$

and $n: \mathbb{R} \rightarrow \mathbb{R}$ given by

$$
n(x)=\left\{\begin{array}{l}
0, \text { if } x=1 \\
x-2, \text { if } x \neq 1
\end{array}\right.
$$

we have that

$$
\underset{n(x) \rightarrow n(1)}{\operatorname{glim}} f(x)=3=f(1),
$$

but $n^{-1}(\{n(1)\})=\{1,2\} \neq\{1\}$. Still, $f$ is $n$-continuous at 1 , because given $\epsilon>0$ and taking $\delta=\min \{1, \epsilon\}$, then the condition (7) is satisfied, this is, although $|n(2)-n(1)|=$ 0 , we still have that $|f(2)-f(1)|=0$. However, note that $f$ is not continuous at 1 in the usual sense.

The previous example highlights that generalized continuity and usual continuity are not equivalent definitions. Another important observation resulting from the previous example is that the condition $n^{-1}(\{n(a)\})=\{a\}$ in Theorem 1 (iii) is not a necessary condition for generalized continuity.

Lemma 1. Let $X$ be a non-empty set, let $\left(Y, d_{Y}\right)$ and $\left(Z, d_{Z}\right)$ be metric spaces, let $f: X \rightarrow\left(Y, d_{Y}\right)$ and $n: X \rightarrow\left(Z, d_{Z}\right)$ be functions and let $a \in X$. The function $f$ is n-continuous at a if, and only if, for every $\epsilon>0$, there exists $a \delta>0$ such that

$$
f\left(n^{-1}\left(B_{Z}(n(a) ; \delta)\right)\right) \subset B_{Y}(f(a) ; \epsilon) .
$$

Demonstração. Let $\epsilon>0$. Since $f$ is $n$-continuous at $a$, it follows that for this $\epsilon>0$, there exists a $\delta>0$ such that

$$
x \in X \text { and } d_{Z}(n(x), n(a))<\delta \text { implies } d_{Y}(f(x), f(a))<\epsilon .
$$


Let $y \in f\left(n^{-1}\left(B_{Z}(n(a) ; \delta)\right)\right)$. Then there exists $x \in n^{-1}\left(B_{Z}(n(a) ; \delta)\right)$ such that $f(x)=$ $y$. If $x \in n^{-1}\left(B_{Z}(n(a) ; \delta)\right)$, then $n(x) \in B_{Z}(n(a) ; \delta)$. Hence, having $d_{Z}(n(x), n(a))<$ $\delta$, it follow that $d_{Y}(f(x), f(a))<\epsilon$, which is equivalent to $y=f(x) \in B_{Y}(f(a) ; \epsilon)$. Therefore, $f\left(n^{-1}\left(B_{Z}(n(a) ; \delta)\right)\right) \subset B_{Y}(f(a) ; \epsilon)$.

Conversely, let $a \in X$ and let $\epsilon>0$. By hypothesis, for this $\epsilon>0$, there exists a $\delta>0$ such that $f\left(n^{-1}\left(B_{Z}(n(a) ; \delta)\right)\right) \subset B_{Y}(f(a) ; \epsilon)$. Hence, if $x \in X$ and $d_{Z}(n(x), g(a))<\delta$, then $n(x) \in B_{Z}(n(a) ; \delta)$ and $x \in n^{-1}\left(B_{Z}(n(a) ; \delta)\right)$. Thus, $f(x) \in f\left(n^{-1}\left(B_{Z}(n(a) ; \delta)\right)\right) \subset$ $B_{Y}(f(a) ; \epsilon)$, resulting in $d_{Y}(f(x), f(a))<\epsilon$. Therefore, $f$ is $n$-continuous at $a$.

Lemma 2. Let $X$ be a non-empty set, let $\left(Y, d_{Y}\right)$ and $\left(Z, d_{Z}\right)$ be metric spaces, let $f: X \rightarrow\left(Y, d_{Y}\right)$ and $n: X \rightarrow\left(Z, d_{Z}\right)$ be functions and let $a \in X$. The following statements hold true:

(i) If $f$ is n-continuous at a, then for every $\epsilon>0$, there exists $a \delta>0$ such that

$$
B_{Z}(n(a) ; \delta) \cap n(X) \subset n\left(f^{-1}\left(B_{Y}(f(a) ; \epsilon)\right)\right)
$$

(ii) If $n$ is injective and for every $\epsilon>0$, there exists $a \delta>0$ such that

$$
B_{Z}(n(a) ; \delta) \cap n(X) \subset n\left(f^{-1}\left(B_{Y}(f(a) ; \epsilon)\right)\right)
$$

then $f$ is n-continuous at a.

Demonstração. (i) Let $\epsilon>0$. Since $f$ is $n$-continuous at $a$, it follows from Lemma 1 that for this $\epsilon>0$, there exists a $\delta>0$ such that

$$
f\left(n^{-1}\left(B_{Z}(n(a) ; \delta)\right)\right) \subset B_{Y}(f(a) ; \epsilon) .
$$

According to Proposition 4 (iii), the expression (8) is equivalent to

$$
n^{-1}\left(B_{Z}(n(a) ; \delta)\right) \subset f^{-1}\left(B_{Y}(f(a) ; \epsilon)\right)
$$

It follows from Proposition 4 (i) that

$$
B_{Z}(n(a) ; \delta) \cap n(X)=n\left(n^{-1}\left(B_{Z}(n(a) ; \delta)\right) \cap X\right)=n\left(n^{-1}\left(B_{Z}(n(a) ; \delta)\right)\right)
$$

Using (10) and Proposition 1(ii) in (9), it results that

$$
B_{Z}(n(a) ; \delta) \cap n(X)=n\left(n^{-1}\left(B_{Z}(n(a) ; \delta)\right)\right) \subset n\left(f^{-1}\left(B_{Y}(f(a) ; \epsilon)\right)\right)
$$


Therefore, for every $\epsilon>0$, there exists a $\delta>0$ such that

$$
B_{Z}(n(a) ; \delta) \cap n(X) \subset n\left(f^{-1}\left(B_{Y}(f(a) ; \epsilon)\right)\right)
$$

(ii) Let $\epsilon>0$. By hypothesis, for this $\epsilon>0$, there exists a $\delta>0$ such that

$$
B_{Z}(n(a) ; \delta) \cap n(X) \subset n\left(f^{-1}\left(B_{Y}(f(a) ; \epsilon)\right)\right)
$$

It results from (10) and (11) that

$$
n\left(n^{-1}\left(B_{Z}(n(a) ; \delta)\right)\right) \subset n\left(f^{-1}\left(B_{Y}(f(a) ; \epsilon)\right)\right) .
$$

According to Proposition 4 (iii), the expression (12) is equivalent to

$$
n^{-1}\left(B_{Z}(n(a) ; \delta)\right) \subset n^{-1}\left(n\left(f^{-1}\left(B_{Y}(f(a) ; \epsilon)\right)\right)\right) .
$$

Since $n$ is injective by hypothesis, it follows from Proposition 3 (ii) that

$$
n^{-1}\left(n\left(f^{-1}\left(B_{Y}(f(a) ; \epsilon)\right)\right)\right)=f^{-1}\left(B_{Y}(f(a) ; \epsilon)\right) .
$$

It results from (13) and (14) that

$$
n^{-1}\left(B_{Z}(n(a) ; \delta)\right) \subset f^{-1}\left(B_{Y}(f(a) ; \epsilon)\right)
$$

and using Proposition 4 (iii), then $(15)$ is equivalent to

$$
f\left(n^{-1}\left(B_{Z}(n(a) ; \delta)\right)\right) \subset B_{Y}(f(a) ; \epsilon) .
$$

It results from (16) and Lemma 1 that $f$ is $n$-continuous at $a$.

Theorem 2. Let $X$ be a non-empty set, let $\left(Y, d_{Y}\right)$ and $\left(Z, d_{Z}\right)$ be metric spaces, let $f: X \rightarrow\left(Y, d_{Y}\right)$ and $n: X \rightarrow\left(Z, d_{Z}\right)$ be functions and let $a \in X$. The function $f$ is $n$-continuous at a if, and only if, for every open subset $U$ of $Y$ (in metric sense) containing $f(a)$, there exists an open subset $V$ of $Z$ (in metric sense) containing $n(a)$ such that

$$
f\left(n^{-1}(V)\right) \subset U
$$

Demonstração. Let $U$ be an open subset of $Y$ such that $f(a) \in U$. If $U$ is an open subset of $Y$, then $f(a)$ is an interior point of $U$ and consequently there exists $\epsilon>0$ such that

$$
B_{Y}(f(a) ; \epsilon) \subset U .
$$


Since $f$ is $n$-continuous at $a$, it follows from Lemma 1 that for this $\epsilon>0$, there exists a $\delta>0$ such that

$$
f\left(n^{-1}\left(B_{Z}(n(a) ; \delta)\right)\right) \subset B_{Y}(f(a) ; \epsilon) .
$$

Taking $V=B_{Z}(n(a) ; \delta)$, we have that $V$ is an open subset of $Z$ containing $n(a)$ and it results from (17) and (18) that

$$
f\left(n^{-1}(V)\right) \subset B_{Y}(f(a) ; \epsilon) \subset U
$$

Conversely, let $a \in X$ and $\epsilon>0$. Since the open ball $B_{Y}(f(a) ; \epsilon)$ is an open subset of $Y$ containing $f(a)$, it follows from the hypothesis that there exists an open subset $V$ of $Z$ containing $n(a)$ such that

$$
f\left(n^{-1}(V)\right) \subset B_{Y}(f(a) ; \epsilon) .
$$

If $V$ is open subset of $Z$, then $n(a)$ is an interior point of $V$ and consequently there exists a $\delta>0$ such that

$$
B_{Z}(n(a) ; \delta) \subset V
$$

Using Proposition 2 (i) and Proposition 1 (ii) consecutively in 20 and combining with (19), we have that

$$
f\left(n^{-1}\left(B_{Z}(n(a) ; \delta)\right)\right) \subset f\left(n^{-1}(V)\right) \subset B_{Y}(f(a) ; \epsilon) .
$$

It results from (21) and Lemma 1 that $f$ is $n$-continuous at $a$.

Theorem 3. Let $X$ be a non-empty set, let $\left(Y, d_{Y}\right)$ and $\left(Z, d_{Z}\right)$ be metric spaces, let $f: X \rightarrow\left(Y, d_{Y}\right)$ and $n: X \rightarrow\left(Z, d_{Z}\right)$ be functions and let $a \in X$. The following statements hold true:

(i) If $f$ is n-continuous at a, then for every open subset $U$ of $Y$ (in metric sense) containing $f(a)$, there exists an open subset $V$ of $Z$ (in metric sense) containing $n(a)$ such that

$$
\left.V \cap n(X) \subset n\left(f^{-1}(U)\right)\right)
$$

(ii) If $n$ is injective and for every open subset $U$ of $Y$ (in metric sense) containing $f(a)$, there exists an open subset $V$ of $Z$ (in metric sense) containing $n(a)$ such that

$$
\left.V \cap n(X) \subset n\left(f^{-1}(U)\right)\right)
$$


then $f$ is n-continuous at a.

Demonstração. (i) Let $U$ be an open subset of $Y$ such that $f(a) \in U$. Thus, there exists $\epsilon>0$ such that

$$
B_{Y}(f(a) ; \epsilon) \subset U
$$

Since $f$ is $n$-continuous in $a$, it follows from Lemma 2 that for this $\epsilon>0$, there exists a $\delta>0$ such that

$$
B_{Z}(n(a) ; \delta) \cap n(X) \subset n\left(f^{-1}\left(B_{Y}(f(a) ; \epsilon)\right)\right)
$$

Taking $V=B_{Z}(n(a) ; \delta)$, we have that $V$ is an open subset of $Z$ containing $n(a)$ and using Proposition 2 (i) and Proposition 1 (ii) consecutively in (22) together with (23) we have that

$$
V \cap n(X) \subset n\left(f^{-1}\left(B_{Y}(f(a) ; \epsilon)\right)\right) \subset n\left(f^{-1}(U)\right)
$$

(ii) Let $\epsilon>0$. Since the open ball $B_{Y}(f(a) ; \epsilon)$ is an open subset of $Y$ containing $f(a)$, it follows from hypothesis that there exists an open subset $V$ of $Z$ containing $n(a)$ such that

$$
V \cap n(X) \subset n\left(f^{-1}\left(B_{Y}(f(a) ; \epsilon)\right)\right)
$$

Since $V$ is open there exists $\delta>0$ such that

$$
B_{Z}(n(a) ; \delta) \subset V .
$$

It follows from (24) and (25) that

$$
B_{Z}(n(a) ; \delta) \cap n(X) \subset V \cap n(X) \subset n\left(f^{-1}\left(B_{Y}(f(a) ; \epsilon)\right)\right) .
$$

Thus, using (26), the hypothesis that $n$ is injective and Lemma 2 (ii) results that $f$ is $n$-continuous at $a$.

\section{Generalized continuity in topological context}

Now, based on Theorem 2 and Theorem 3 are proposed the following three definitions in the context of functions between topological spaces.

Definition 9. Let $X$ be a non-empty set, let $\left(Y, \mathcal{T}^{Y}\right)$ and $\left(Z, \mathcal{T}^{Z}\right)$ be topological spaces, let $f: X \rightarrow\left(Y, \mathcal{T}^{Y}\right)$ and $n: X \rightarrow\left(Z, \mathcal{T}^{Z}\right)$ be functions and let $a \in X$. The function $f$ is 
said to be $n$-continuous at a iffor every $U \in \mathcal{T}_{f(a)}^{Y}$, there exists $a V \in \mathcal{T}_{n(a)}^{Z}$ such that

$$
f\left(n^{-1}(V)\right) \subset U
$$

In this text the set of all functions from a set $A$ to a topological space $(B, \mathcal{T})$ shall be denoted by $\mathrm{F}(A, B, \mathcal{T})$. Fixed a function of $n: X \rightarrow\left(Z, \mathcal{T}^{Z}\right)$, which from now on will be named only by continuator, and fixed a topological space $\left(Y, \mathcal{T}^{Y}\right)$, then the set of all functions from $X$ to $\left(Y, \mathcal{T}^{Y}\right)$ that are $n$-continuous at $a \in X$ shall be denoted by $\mathrm{C}_{n}(a, X, Y)$, that is,

$$
\mathrm{C}_{n}(a, X, Y)=\left\{f \in \mathrm{F}\left(X, Y, \mathcal{T}^{Y}\right): f \text { is } n \text {-continuous at } a\right\} \text {. }
$$

If we fix now a function $f: X \rightarrow\left(Y, \mathcal{T}^{Y}\right)$, which from now on will be named only by continuant, and fixed a topological space $\left(Z, \mathcal{T}^{Z}\right)$, one can consider the set

$$
\mathrm{C}^{f}(a, X, Z)=\left\{n \in \mathrm{F}\left(X, Z, \mathcal{T}^{Z}\right): f \in \mathrm{C}_{n}(a, X, Y)\right\}
$$

It should be noted that in 28 ) and 29$]$ the topologies in the codomains of the functions are omitted in the notations of the sets, since this information is clear in the context.

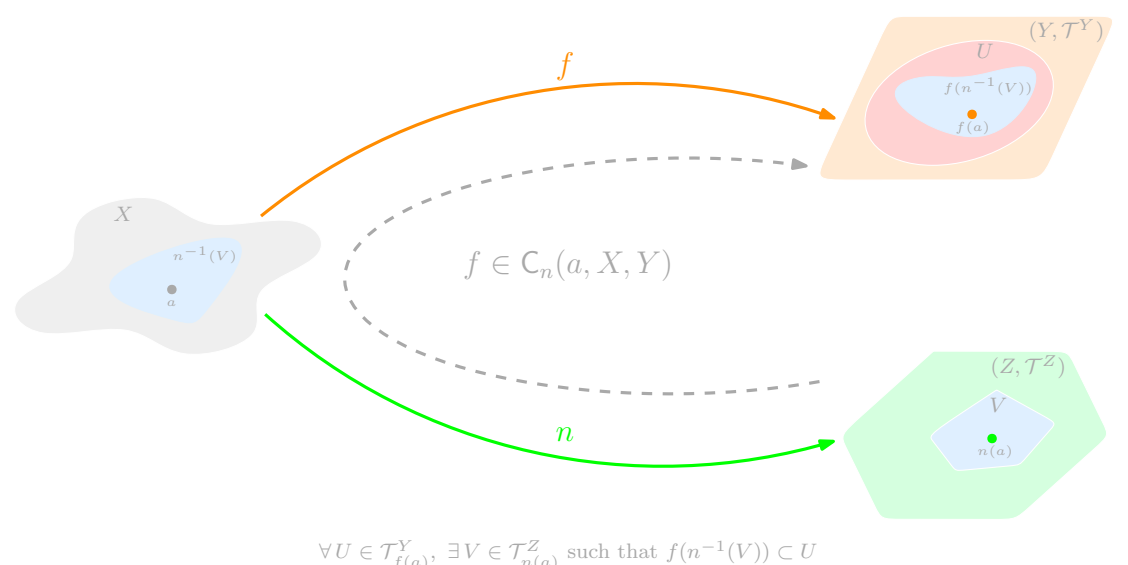

Figure 2: generalized continuity

Example 4. Let $X$ be a non-empty set, let $\left(Y, \mathcal{T}^{Y}\right)$ be topological space and let a $\in X$. We have that any $f: X \rightarrow\left(Y, \mathcal{T}^{Y}\right)$ is $f$-continuous at a. Indeed, given $U \in \mathcal{T}_{f(a)}^{Y}$, then take $V=U$ and it follows from Proposition 3 (iv) that

$$
f\left(f^{-1}(V)\right)=f\left(f^{-1}(U)\right) \subset U .
$$


Therefore, $f$ is $f$-continuous at a, that is, $f \in \mathrm{C}_{f}(a, X, Y)$ and $f \in \mathbf{C}^{f}(a, X, Y)$.

Example 5. Let $\left(X, \mathcal{T}^{X}\right)$ and $\left(Y, \mathcal{T}^{Y}\right)$ be topological spaces, let $f:\left(X, \mathcal{T}^{X}\right) \rightarrow\left(Y, \mathcal{T}^{Y}\right)$, $i d:\left(X, \mathcal{T}^{X}\right) \rightarrow\left(X, \mathcal{T}^{X}\right)$ be functions and let $a \in X$. We have that $f$ is continuous at $a$ if, and only if, $f$ is id-continuous at a. Indeed, when considered a $V \in \mathcal{T}_{a}^{X}$, just note that $f\left(i d^{-1}(V)\right)=f(V)$ to conclude, in this case, that the conditions (3) and (27) are equivalent.

Example 6. Let $\mathbb{R}$ be equipped with usual topology, let $a>0$ be a real number and let $i d: \mathbb{R} \rightarrow \mathbb{R}$ be the identity function and $m: \mathbb{R} \rightarrow \mathbb{R}$ the function given by $m(x)=|x|$. For every open subset $V$ of $\mathbb{R}$ containing $m(a)=a$, consider $V^{+}=V \cap[0, \infty)$ and $V^{-}=\left\{x \in \mathbb{R}:-x \in V^{+}\right\}$. Note that $m^{-1}(V)=V^{+} \cup V^{-}$. Thus, given the open subset $U=(0, \infty)$ of $\mathbb{R}$ containing $i d(a)=a$, it results that for every open subset $V$ of $\mathbb{R}$ containing $m(a)=a$ is valid that

$$
i d\left(m^{-1}(V)\right)=i d\left(V^{+} \cup V^{-}\right)=V^{+} \cup V^{-} \not \subset(0, \infty)=U .
$$

Therefore, $i d$ is not $m$-continuous at a, that is, $i d \notin \mathrm{C}_{m}(a, \mathbb{R}, \mathbb{R})$ and $m \notin \mathbf{C}^{i d}(a, \mathbb{R}, \mathbb{R})$.

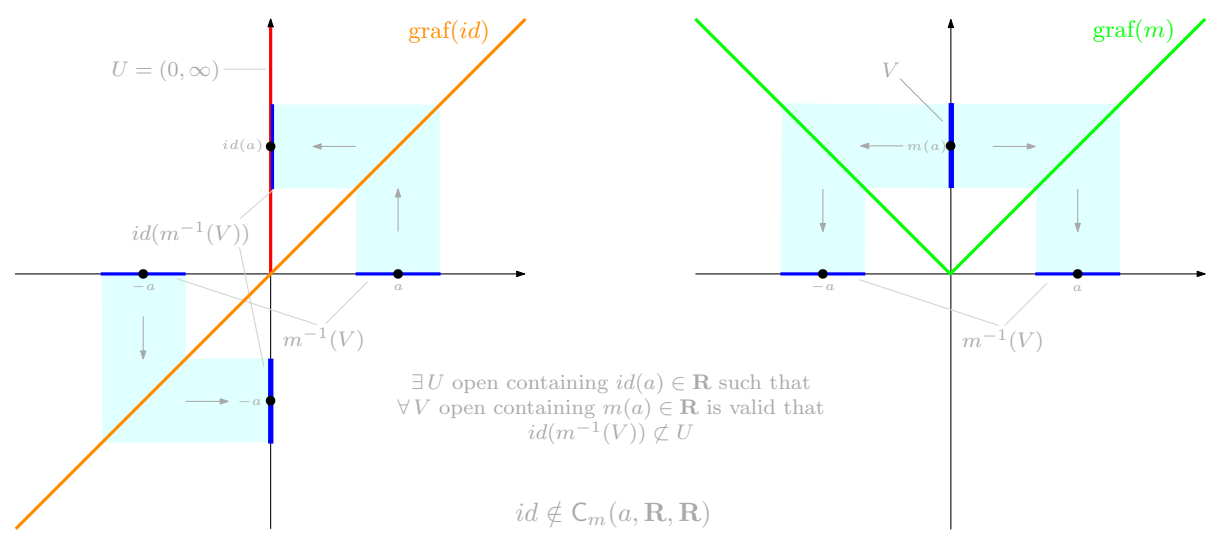

Figure 3: an example of non-generalized continuity

Proposition 13. Let $X$ be a non-empty set, let $\left(Y, \mathcal{T}^{Y}\right)$ and $\left(Z, \mathcal{T}^{Z}\right)$ be topological spaces, let $f: X \rightarrow\left(Y, \mathcal{T}^{Y}\right)$ and $n: X \rightarrow\left(Z, \mathcal{T}^{Z}\right)$ be functions and let $a \in X$. The following statements hold true:

(i) If $f$ is constant function, then $f \in \mathrm{C}_{n}(a, X, Y)$.

(ii) If $n$ is constant function and $f \in \mathrm{C}_{n}(a, X, Y)$, then $f(X) \subset U$, for all $U \in \mathcal{T}_{f(a)}^{Y}$. 
Demonstração. (i) Let $U \in \mathcal{T}_{f(a)}^{Y}$. Take any $V \in \mathcal{T}_{n(a)}^{Z}$. Note that $f\left(n^{-1}(V)\right)=\{f(a)\}$, because $f$ is a constant function and hence $f\left(n^{-1}(V)\right)=\{f(a)\} \subset U$. Therefore, $f \in \mathrm{C}_{n}(X, Y)$.

(ii) Let $U \in \mathcal{T}_{f(a)}^{Y}$. Since $f \in \mathrm{C}_{n}(a, X, Y)$, then there exists $V \in \mathcal{T}_{n(a)}^{Z}$ such that $f\left(n^{-1}(V)\right) \subset U$. On the other hand, since $n$ is constant function and $n(a) \in V$, then $n^{-1}(V)=X$. Thus, $f(X)=f\left(n^{-1}(V)\right) \subset U$. Therefore, $f(X) \subset U$, for all $U \in \mathcal{T}_{f(a)}^{Y}$.

Definition 10. Let $X$ be a non-empty set, let $\left(Y, \mathcal{T}^{Y}\right)$ and $\left(Z, \mathcal{T}^{Z}\right)$ be topological spaces, let $f: X \rightarrow\left(Y, \mathcal{T}^{Y}\right)$ and $n: X \rightarrow\left(Z, \mathcal{T}^{Z}\right)$ be functions and let $a \in X$. The function $f$ is said to be widely $n$-continuous at a if for every $U \in \mathcal{T}_{f(a)}^{Y}$, there exists a $V \in \mathcal{T}_{n(a)}^{Z}$ such that

$$
V \cap n(X) \subset n\left(f^{-1}(U)\right)
$$

The set of all functions from $X$ to $\left(Y, \mathcal{T}^{Y}\right)$ that are widely $n$-continuous at $a$ is denoted by $\mathrm{W}_{n}(a, X, Y)$, that is,

$$
\mathrm{W}_{n}(a, X, Y)=\left\{f \in \mathrm{F}\left(X, Y, \mathcal{T}^{Y}\right): f \text { is widely } n \text {-continuous at } a\right\}
$$

Fixed an continuant $f: X \rightarrow\left(Y, \mathcal{T}^{Y}\right)$ and fixed a topological space $\left(Z, \mathcal{T}^{Z}\right)$, one can consider the set

$$
\mathbf{W}^{f}(a, X, Z)=\left\{n \in \mathrm{F}\left(X, Z, \mathcal{T}^{Z}\right): f \in \mathbf{W}_{n}(a, X, Y)\right\}
$$

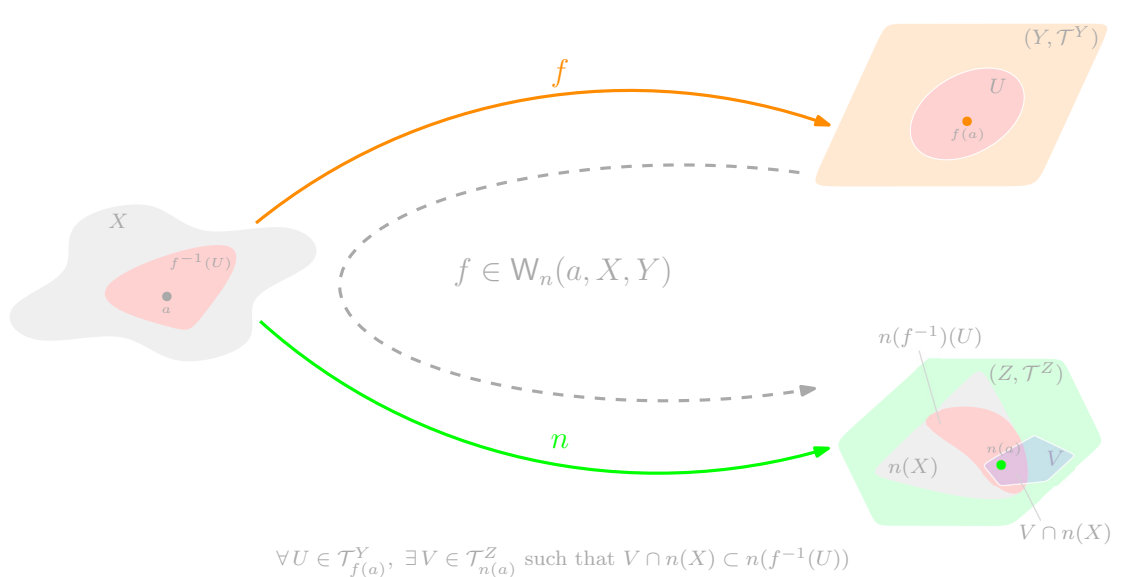

Figure 4: generalized continuity in the wide sense 
Example 7. Let $X$ be a non-empty set, let $\left(Y, \mathcal{T}^{Y}\right)$ be topological space and let $a \in X$. We have that any $f: X \rightarrow\left(Y, \mathcal{T}^{Y}\right)$ is widely $f$-continuous at a. Indeed, given $U \in \mathcal{T}_{f(a)}^{Y}$, then take $V=U$ and it follows from Proposition 4(i) that

$$
V \cap f(X)=U \cap f(X)=f\left(f^{-1}(U) \cap X\right)=f\left(f^{-1}(U)\right) .
$$

Therefore, $f$ is widely $f$-continuous at a, that is, $f \in \mathbf{W}_{f}(a, X, Y)$ and $f \in \mathbf{W}^{f}(a, X, Y)$.

Example 8. Let $\mathbb{R}$ be equipped with usual topology, let $a>0$ be a real number and let $i d: \mathbb{R} \rightarrow \mathbb{R}$ be the identity function and $m: \mathbb{R} \rightarrow \mathbb{R}$ the function given by $m(x)=|x|$. Given $U$ a open subset of $\mathbb{R}$ containing $i d(a)=a$, then $V=U \cap(0, \infty)$ is a open subset of $\mathbb{R}$ containing $m(a)=a$ and $V \subset m(U)$. Thus, it is valid that

$$
V \cap m(\mathbb{R})=V \cap[0, \infty)=V \subset m(U)=m\left(i d^{-1}(U)\right)
$$

Hence, $i d$ is widely $m$-continuous at a, that is, $i d \in \mathbf{W}_{m}(a, \mathbb{R}, \mathbb{R})$ and $m \in \mathbf{W}^{i d}(a, \mathbb{R}, \mathbb{R})$.

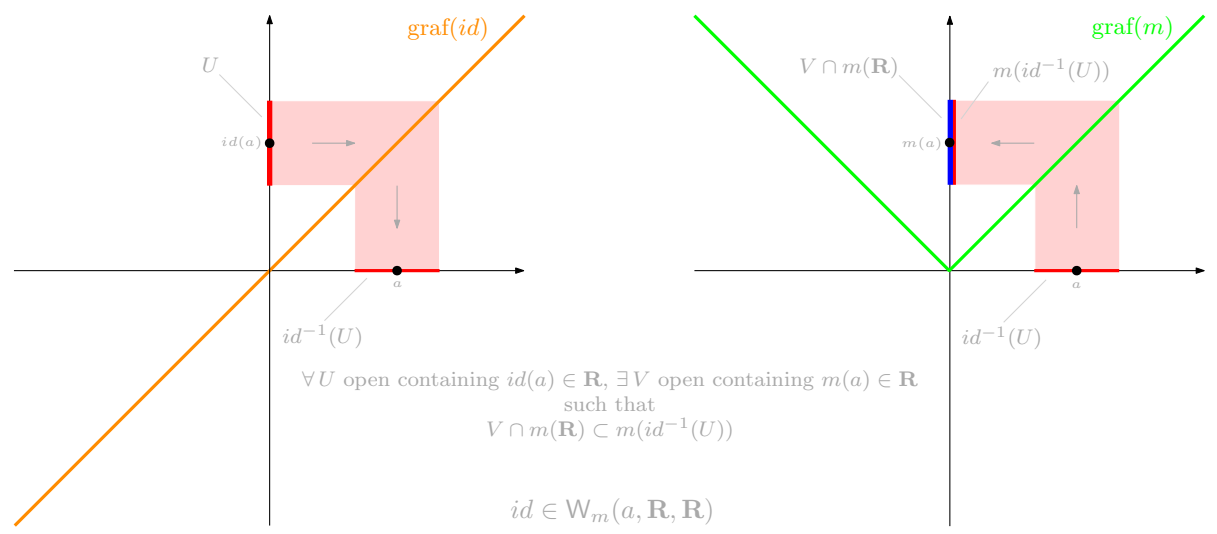

Figure 5: an example of generalized continuity in the wide sense

Proposition 14. Let $X$ be a non-empty set, let $\left(Y, \mathcal{T}^{Y}\right)$ and $\left(Z, \mathcal{T}^{Z}\right)$ be topological spaces, let $f: X \rightarrow\left(Y, \mathcal{T}^{Y}\right)$ and $n: X \rightarrow\left(Z, \mathcal{T}^{Z}\right)$ be functions and let $a \in X$. If $n$ is a constant function, then $f \in \mathrm{W}_{n}(a, X, Y)$.

Demonstração. Let $U \in \mathcal{T}_{f(a)}^{Y}$. Since $n$ is constant function, then $n(X)=\{n(a)\}=$ $n\left(f^{-1}(U)\right)$. Taking any $V \in \mathcal{T}_{n(a)}^{Z}$, we have that $V \cap n(X)=\{n(a)\}=n\left(f^{-1}(U)\right)$. Therefore, $f \in \mathrm{W}_{n}(a, X, Y)$. 
Definition 11. Let $X$ be a non-empty set, let $\left(Y, \mathcal{T}^{Y}\right)$ and $\left(Z, \mathcal{T}^{Z}\right)$ be topological spaces, let $f: X \rightarrow\left(Y, \mathcal{T}^{Y}\right)$ and $n: X \rightarrow\left(Z, \mathcal{T}^{Z}\right)$ be functions and let $a \in X$. The function $f$ is said to be strictly $n$-continuous at a if $n$ is injective and for every $U \in \mathcal{T}_{f(a)}^{Y}$, there exists $a V \in \mathcal{T}_{n(a)}^{Z}$ such that

$$
V \cap n(X) \subset n\left(f^{-1}(U)\right)
$$

The set of all functions from $X$ to $\left(Y, \mathcal{T}^{Y}\right)$ that are strictly $n$-continuous at $a$, if $\mathrm{n}$ is infective, is denoted by $\mathrm{S}_{n}(a, X, Y)$, that is,

$$
\mathrm{S}_{n}(a, X, Y)=\left\{f \in \mathrm{F}\left(X, Y, \mathcal{T}^{Y}\right): f \text { is strictly } n \text {-continuous at } a\right\} \text {. }
$$

Fixed an continuant $f: X \rightarrow\left(Y, \mathcal{T}^{Y}\right)$ and fixed a topological space $\left(Z, \mathcal{T}^{Z}\right)$, one can consider the set

$$
\mathbf{S}^{f}(a, X, Z)=\left\{n \in \mathrm{F}\left(X, Z, \mathcal{T}^{Z}\right): n \text { is injective and } f \in \mathrm{W}_{n}(a, X, Y)\right\}
$$

Theorem 4. Let $X$ be a non-empty set, let $\left(Y, \mathcal{T}^{Y}\right)$ and $\left(Z, \mathcal{T}^{Z}\right)$ be topological spaces, let $f: X \rightarrow\left(Y, \mathcal{T}^{Y}\right)$ and $n: X \rightarrow\left(Z, \mathcal{T}^{Z}\right)$ be functions and let $a \in X$. The following statements hold true:

(i) If $f$ is n-continuous at a, then $f$ is widely $n$-continuous at $a$.

(ii) If $f$ strictly $n$-continuous at a, then $f$ is $n$-continuous at $a$.

Demonstração. (i) Let $\mathcal{U} \in \mathcal{T}_{f(a)}^{Y}$. Since $f$ is $n$-continuous at $a$, then there exists a $V \in$ $\mathcal{T}_{n(a)}^{Z}$ such that $f\left(n^{-1}(V)\right) \subset U$ and using Proposition 4 (iii) this inclusion is equivalent to

$$
n^{-1}(V) \subset f^{-1}(U)
$$

Taking this $V \in \mathcal{T}_{n(a)}^{Z}$, it follows from Proposition 4 (i) that

$$
V \cap n(X)=n\left(n^{-1}(V) \cap X\right)=n\left(n^{-1}(V)\right) .
$$

Using Proposition 1(ii), with the function $n$, in (31) and combining the result with (32), we have to

$$
V \cap n(X)=n\left(n^{-1}(V)\right) \subset n\left(f^{-1}(U)\right)
$$

Therefore, $f$ is widely $n$-continuous at $a$. 
(ii) Let $\mathcal{U} \in \mathcal{T}_{f(a)}^{Y}$. Since $f$ is strictly $n$ - continuous at $a$ by hypothesis, then there exists a $V \in \mathcal{T}_{n(a)}^{Z}$ such that $V \cap n(X) \subset n\left(f^{-1}(U)\right)$, which is equivalent according to expression (32) to

$$
n\left(n^{-1}(V)\right) \subset n\left(f^{-1}(U)\right)
$$

According to Proposition 4 (iii), the expression (33) is equivalent to

$$
n^{-1}(V) \subset n^{-1}\left(n\left(f^{-1}(U)\right)\right)
$$

Since $n$ is injective, since $f$ is strictly $n$-continuous at $a$ by hypothesis, it follows from Proposition 3 (ii) that

$$
n^{-1}\left(n\left(f^{-1}(U)\right)\right)=f^{-1}(U)
$$

It results from (34) and (35) that $n^{-1}(V) \subset f^{-1}(U)$ and using Proposition 4 (iii), this inclusion is equivalent to

$$
f\left(n^{-1}(V)\right) \subset U
$$

Therefore, $f$ is $n$-continuous at $a$.

Corollary 1. Let $X$ be a non-empty set, let $\left(Y, \mathcal{T}^{Y}\right)$ and $\left(Z, \mathcal{T}^{Z}\right)$ be topological spaces, let $f: X \rightarrow\left(Y, \mathcal{T}^{Y}\right)$ and $n: X \rightarrow\left(Z, \mathcal{T}^{Z}\right)$ be functions and let $a \in X$. If $f$ is a constant function, then $f \in \mathrm{W}_{n}(a, X, Y)$.

Demonstração. It follows from Proposition 13 (i) and Theorem 4 (i).

From Theorem 4 we conclude that $\mathrm{C}_{n}(a, X, Y) \subset \mathrm{W}_{n}(a, X, Y)$ and if $n$ is injective we also have that $\mathrm{S}_{n}(a, X, Y) \subset \mathrm{C}_{n}(a, X, Y)$. On the other hand, $\mathrm{S}_{n}(a, X, Y)=$ $\mathrm{W}_{n}(a, X, Y)$ by definition, in the case that $n$ is injective. In summary, for every continuator $n$ is valid that

$$
\mathrm{C}_{n}(a, X, Y) \subset \mathrm{W}_{n}(a, X, Y)
$$

and if $n$ is a injective continuator, then is valid that

$$
\mathrm{S}_{n}(a, X, Y)=\mathrm{C}_{n}(a, X, Y)=\mathrm{W}_{n}(a, X, Y),
$$

that is, for injective continuators the concepts of generalized continuity and wide generalized continuity are coincident. 
Also it follows from Theorem 4 that for any continuant $f$ fixed is valid that

$$
\mathbf{S}^{f}(a, X, Z) \subset \mathbf{C}^{f}(a, X, Z) \subset \mathbf{W}^{f}(a, X, Z)
$$

Example 9. We already know that any continuous function at a point a is id-continuous at $a$ and hence, from Theorem 4 is also widely id-continuous at a and consequently strictly id-continuous at a, since id is injective.

Example 10. Consider a set $X=\{a, b\}$, with $a \neq b$, equipped with the Sierpinski topology $\mathcal{S}=\{\emptyset,\{a\}, X\}$. Consider also the identity function $i d: X \rightarrow(X, \mathcal{S})$ given by $i d(a)=a$ and $i d(b)=b$ and consider the permutation function $p: X \rightarrow(X, \mathcal{S})$ given by $p(a)=b$ and $p(b)=a$. Note that $\mathcal{S}_{p(b)}=\mathcal{S}_{i d(a)}=\mathcal{S}_{a}=\{\{a\}, X\}$ and $\mathcal{S}_{p(a)}=\mathcal{S}_{i d(b)}=\mathcal{S}_{b}=\{X\}$. Since $U=X$ is unique in $\mathcal{S}_{i d(b)}$, take $V=\{a\} \in \mathcal{S}_{p(b)}$ and hence

$$
i d\left(p^{-1}(V)\right)=i d\left(p^{-1}(\{a\})\right)=\{b\} \subset X=U .
$$

Therefore, id is p-continuous at $b$ and consequently id is widely p-continuous at $b$. Moreover, taking $U=\{a\} \in \mathcal{S}_{i d(a)}$, then there exist only $V=X \in \mathcal{S}_{p(a)}$ and

$$
V \cap p(X)=X \not \subset\{b\}=p(\{a\})=p\left(i d^{-1}(U)\right)
$$

Therefore id is not widely p-continuous at a and consequently id is not p-continuous at a. Note that if $p \notin \mathbf{W}^{i d}(a, X, X)$, then $p \notin \mathbf{S}^{i d}(a, X, X)$, although $p$ is injective.

Now, we will present examples for which the inclusions given in (36) and (37) are proper inclusions.

Example 11. Let $\mathbb{R}$ be equipped with usual topology, let $a \in \mathbb{R}_{+}$and let $i d: \mathbb{R} \rightarrow \mathbb{R}$ be the identity function and $m: \mathbb{R} \rightarrow \mathbb{R}$ the function given by $m(x)=|x|$. As seen in Examples 8 and 6, we have that id is widely m-continuous at a, but id is not m-continuous at a. In summary, $\mathrm{C}_{m}(a, \mathbb{R}, \mathbb{R}) \varsubsetneqq \mathrm{W}_{m}(a, \mathbb{R}, \mathbb{R})$ and $\mathbf{C}^{i d}(a, \mathbb{R}, \mathbb{R}) \varsubsetneqq \mathbf{W}^{i d}(a, \mathbb{R}, \mathbb{R})$.

Example 12. Let $\mathbb{R}$ be equipped with usual topology, $a \in \mathbb{R}_{+}$and let $m: \mathbb{R} \rightarrow \mathbb{R}$ be the function given by $m(x)=|x|$. Note that $m$ is widely $m$-continuous at a and $m$ is $m$-continuous at a, but $m$ is not strictly $m$-continuous at a, because $m$ is not injective. Thus, $m \notin \mathbf{S}^{m}(a, \mathbb{R}, \mathbb{R})$, but $m \in \mathbf{C}^{m}(a, \mathbb{R}, \mathbb{R})$. Therefore, $\mathbf{S}^{m}(a, \mathbb{R}, \mathbb{R}) \varsubsetneqq \mathbf{C}^{m}(a, \mathbb{R}, \mathbb{R})$.

To assist the next theorem, we present requirements related to nets (see [1] and [13]).

Definition 12. A set $\Lambda$, equipped with a order relation $\leq$, is called directed set if the following conditions are satisfied: 
(a) $\lambda \leq \lambda$, for all $\lambda \in \Lambda$.

(b) Given $\lambda, \mu, \nu \in \Lambda$ such that $\lambda \leq \mu$ and $\mu \leq \nu$, then $\lambda \leq \nu$.

(c) Given $\lambda, \mu \in \Lambda$, there exists $\nu \in \Lambda$ such that $\lambda \leq \nu$ and $\mu \leq \nu$.

A directed set $\Lambda$ equipped with a order relation $\leq$ is denoted in this text by $(\Lambda, \leq)$.

Example 13. The set of natural numbers $\mathbb{N}$ equipped with the usual order relation is a directed set.

Example 14. Let $(X, \mathcal{T})$ be a non-empty topological space and let $a \in X$. Consider the order relation on $\mathcal{P}(X)$ given by

$$
A \leq B \text { if, and only if, } A \supset B .
$$

The open neighbourhood basis $\mathcal{T}_{a}=\{U \in \mathcal{T}: a \in U\}$ equipped with order relation $\leq$ given by (38) is a directed set.

Definition 13. Let $X$ be a non-empty set and let $(\Lambda, \leq)$ be a directed set. Any function of form $x:(\Lambda, \leq) \rightarrow X$ is called net from $\Lambda$ to $X$ (or net in $X)$ and denoted by $\left(x_{\lambda}\right)_{\lambda \in \Lambda}$, where $x_{\lambda}=x(\lambda)$.

Definition 14. Let $(X, \mathcal{T})$ be a topological space and let $a \in X$. A net $\left(x_{\lambda}\right)_{\lambda \in \Lambda}$ in $X$ is said to be convergent to a if, for every $U \in \mathcal{T}_{a}$, there exists a $\lambda_{0} \in \Lambda$ such that

$$
\lambda \in \Lambda \text { and } \lambda_{0} \leq \lambda \text { implies } x_{\lambda} \in U \text {. }
$$

A net $\left(x_{\lambda}\right)_{\lambda \in \Lambda}$ in $X$ converging to $a$ is denoted by $x_{\lambda} \rightarrow a$.

Example 15. Every sequence $\left(x_{n}\right)_{n \in \mathbb{N}}$ in $X$ is a net in $X$, and the convergence of nets generalizes the convergence of sequences.

Example 16. Let $(X, \mathcal{T})$ be a topological space and let $a \in X$. Consider $\mathcal{T}_{a}$ equipped with order relation $\leq$ given by (38). If we choose a $x_{U} \in U$, for every $U \in \mathcal{T}_{a}$, then $\left(x_{U}\right)_{U \in \mathcal{T}_{a}}$ is a net in $X$ that converges to $a$.

Theorem 5. Let $X$ be a non-empty set, let $\left(Y, \mathcal{T}^{Y}\right)$ and $\left(Z, \mathcal{T}^{Z}\right)$ be topological spaces, let $f: X \rightarrow\left(Y, \mathcal{T}^{Y}\right)$ and $n: X \rightarrow\left(Z, \mathcal{T}^{Z}\right)$ be functions and let $a \in X$. The function $f$ is n-continuous at a if, and only if, for every net $\left(x_{\lambda}\right)_{\lambda \in \Lambda}$ in $X$, with $n\left(x_{\lambda}\right) \rightarrow n(a)$, it is valid that $f\left(x_{\lambda}\right) \rightarrow f(a)$. 
Demonstraçãoo. Let $\left(x_{\lambda}\right)_{\lambda \in \Lambda}$ a net in $X$ such that $n\left(x_{\lambda}\right) \rightarrow n(a)$. Let $U \in \mathcal{T}_{f(a)}^{Y}$. Since $f \in \mathrm{C}_{n}(a, X, Y)$ by hypothesis, there exists a $V \in \mathcal{T}_{n(a)}^{Z}$ such that $f\left(n^{-1}(V)\right) \subset U$. Since $n\left(x_{\lambda}\right) \rightarrow n(a)$, there exists $\lambda_{0} \in \Lambda$ such that $n\left(x_{\lambda}\right) \in V$, for all $\lambda \geq \lambda_{0}$, which is equivalent to $x_{\lambda} \in n^{-1}(V)$, for all $\lambda \geq \lambda_{0}$. This implies that $f\left(x_{\lambda}\right) \in f\left(n^{-1}(V)\right) \subset U$, for all $\lambda \geq \lambda_{0}$. Therefore, given $U \in \mathcal{T}_{f(a)}^{Y}$, there exists $\lambda_{0} \in \Lambda$ such that $f\left(x_{\lambda}\right) \in U$, for all $\lambda \geq \lambda_{0}$, that is, $f\left(x_{\lambda}\right) \rightarrow f(a)$.

Conversely, we want to show that $f$ is $n$-continuous at $a$. Suppose that $f \notin \mathrm{C}_{n}(a, X, Y)$. Then there exists $U \in \mathcal{T}_{f(a)}^{Y}$ such that $f\left(n^{-1}(V)\right) \not \subset U$, for all $V \in \mathcal{T}_{n(a)}^{Z}$, or equivalently, $n^{-1}(V) \not \subset f^{-1}(U)$, for all $V \in \mathcal{T}_{n(a)}^{Z}$. Thus, $n^{-1}(V) \backslash f^{-1}(U) \neq \emptyset$, for all $V \in \mathcal{T}_{n(a)}^{Z}$. Consider the non-empty family $\mathcal{C}=\left\{n^{-1}(V) \backslash f^{-1}(U) \in \mathcal{P}(X): V \in \mathcal{T}_{n(a)}^{Z}\right\}$. Using Axiom of Choice, there exists a choice function $g: \mathcal{C} \rightarrow \bigcup \mathcal{C}$ such that $g(\mu) \in \mu$, for all $\mu \in \mathcal{C}$. Consider the function $h: \mathcal{T}_{n(a)}^{Z} \rightarrow \mathcal{C}$ given by $h(V)=n^{-1}(V) \backslash f^{-1}(U)$ and note that $\bigcup \mathcal{C} \subset X$. Hence, $x: \mathcal{T}_{n(a)}^{Z} \rightarrow X$ given by $x=g \circ h$ is a net in $X$ such that $x_{V} \in n^{-1}(V) \backslash f^{-1}(U)$, for all $V \in \mathcal{T}_{n(a)}^{Z}$. Since $n\left(x_{V}\right) \in V$, for all $V \in \mathcal{T}_{n(a)}^{Z}$, then $n\left(x_{V}\right) \rightarrow n(a)$, because given $V_{0} \in \mathcal{T}_{n(a)}^{Z}$, it is valid that $n\left(x_{V}\right) \in V_{0}$, for all $V \geq V_{0}$ (or $V \subset V_{0}$ ). On the other hand, $x_{V} \notin f^{-1}(U)$, for all $V \in \mathcal{T}_{n(a)}^{Z}$, which is equivalent to $f\left(x_{V}\right) \notin U$, for all $V \in \mathcal{T}_{n(a)}^{Z}$, and this implies $f\left(x_{V}\right) \not \rightarrow f(a)$. Thus, there exists a net $\left(x_{V}\right)_{V \in \mathcal{T}_{n(a)}^{Z}}$ in $X$ such that $n\left(x_{V}\right) \rightarrow n(a)$, but $f\left(x_{V}\right) \not \rightarrow f(a)$, which contradicts the hypothesis. Therefore, $f$ is $n$-continuous at $a$.

Definition 15. Let $X$ be a non-empty set, let $\left(Y, \mathcal{T}^{Y}\right)$ and $\left(Z, \mathcal{T}^{Z}\right)$ be topological spaces and let $f: X \rightarrow\left(Y, \mathcal{T}^{Y}\right)$ and $n: X \rightarrow\left(Z, \mathcal{T}^{Z}\right)$ be functions. The function $f$ is $n$ continuous (widely $n$-continuous or strictly $n$-continuous) if $f$ is $n$-continuous at a (widely $n$-continuous at a or strictly $n$-continuous at a, respectively), for all $a \in X$.

The set of all functions from $X$ to $\left(Y, \mathcal{T}^{Y}\right)$ that are $n$-continuous (widely $n$-continuous or strictly $n$-continuous) is denoted by $\mathrm{C}_{n}(X, Y)\left(\mathrm{W}_{n}(X, Y)\right.$ or $\mathrm{S}_{n}(X, Y)$, respectively).

Fixed an continuant $f: X \rightarrow\left(Y, \mathcal{T}^{Y}\right)$ and fixed a topological space $\left(Z, \mathcal{T}^{Z}\right)$, we can consider the following sets:

$$
\begin{gathered}
\mathbf{C}^{f}(X, Z)=\left\{n \in \mathrm{F}\left(X, Z, \mathcal{T}^{Z}\right): f \in \mathrm{C}_{n}(X, Y)\right\}=\bigcap_{a \in X} \mathbf{C}^{f}(a, X, Z), \\
\mathbf{W}^{f}(X, Z)=\left\{n \in \mathrm{F}\left(X, Z, \mathcal{T}^{Z}\right): f \in \mathbf{W}_{n}(X, Y)\right\}=\bigcap_{a \in X} \mathbf{W}^{f}(a, X, Z)
\end{gathered}
$$

and

$$
\mathbf{S}^{f}(a, X, Z)=\left\{n \in \mathbf{W}^{f}(X, Z): n \text { is injective }\right\}=\bigcap_{a \in X} \mathbf{S}^{f}(a, X, Z) .
$$


Given a topological space $\left(X, \mathcal{T}^{X}\right)$ and a subset $A$ of $X$, we can equip $A$ with topology $\mathcal{T}^{A}=\left\{U \cap A: U \in \mathcal{T}^{X}\right\}$. The pair $\left(A, \mathcal{T}^{A}\right)$ is called topological subspace of $\left(X, \mathcal{T}^{X}\right)$ and the topology $\mathcal{T}^{A}$ is called induced topology by $\mathcal{T}^{X}$.

Theorem 6. Let $X$ be a non-empty set, let $\left(Y, \mathcal{T}^{Y}\right)$ and $\left(Z, \mathcal{T}^{Z}\right)$ be topological spaces and let $f: X \rightarrow\left(Y, \mathcal{T}^{Y}\right)$ and $n: X \rightarrow\left(Z, \mathcal{T}^{Z}\right)$ be functions. The function $f$ is widely $n$-continuous if, and only if, $n\left(f^{-1}(U)\right) \in \mathcal{T}^{n(X)}$, for all $U \in \mathcal{T}^{Y}$.

Demonstração. Let $U \in \mathcal{T}^{Y}$ and let $z \in n\left(f^{-1}(U)\right)$. Then there exists $a \in f^{-1}(U)$ such that $n(a)=z$ and $f(a) \in U$. Since, by hypothesis, $f \in \mathrm{W}_{n}(X, Y)$ and, in particular, $f$ is widely $n$-continuous at $a$, then there exists $V_{z} \in \mathcal{T}_{n(a)}^{Z}$ such that $V_{z} \cap n(X) \subset n\left(f^{-1}(U)\right)$. Note that $W_{z}=V_{z} \cap n(X)$ is such that $W_{z} \in \mathcal{T}^{n(X)}$. It follows that $n\left(f^{-1}(U)\right)=$ $\bigcup_{z \in n\left(f^{-1}(U)\right)} W_{z}$ and therefore $n\left(f^{-1}(U)\right) \in \mathcal{T}^{n(X)}$.

Conversely, let $a \in X$ and let $U \in \mathcal{T}_{f(a)}^{Y}$. By hypothesis, if follows that $n\left(f^{-1}(U)\right) \in$ $\mathcal{T}^{n(X)}$ and since $\mathcal{T}^{n(X)}$ is a induced topology, then there exists $V \in \mathcal{T}^{Z}$ such that $n\left(f^{-1}(U)\right)=V \cap n(X)$. Note that if $f(a) \in U$, then $a \in f^{-1}(U)$ and $n(a) \in n\left(f^{-1}(U)\right)$, that is, $n(a) \in V$ and $V \in \mathcal{T}_{n(a)}^{Z}$. Thus, given $U \in \mathcal{T}_{f(a)}^{Y}$, there exists $V \in \mathcal{T}_{n(a)}^{Z}$ such that $V \cap n(X)=n\left(f^{-1}(U)\right)$, which is equivalent to $f \in \mathrm{W}_{n}(a, X, Y)$. Since $a$ is arbitrary, then $f \in \mathrm{W}_{n}(a, X, Y)$, for all $a \in X$. Therefore, $f$ is widely $n$-continuous.

Theorem 7. Let $X$ be a non-empty set and let $\left(Y, \mathcal{T}^{Y}\right),\left(Z, \mathcal{T}^{Z}\right)$ and $\left(R, \mathcal{T}^{R}\right)$ be topological spaces. Let $a \in X$ and let $f: X \rightarrow\left(Y, \mathcal{T}^{Y}\right), m: X \rightarrow\left(Z, \mathcal{T}^{Z}\right)$ and $n: X \rightarrow\left(R, \mathcal{T}^{R}\right)$ be functions. The following statements hold true:

(i) If $f \in \mathrm{C}_{m}(a, X, Y)$ and $m \in \mathrm{C}_{n}(a, X, Z)$, then $f \in \mathrm{C}_{n}(a, X, Y)$.

(ii) If $f \in \mathrm{C}_{m}(a, X, Y)$ and $m \in \mathrm{S}_{n}(a, X, Z)$, then $f \in \mathrm{S}_{n}(a, X, Y)$.

(iii) If $f \in \mathrm{C}_{m}(a, X, Y)$ and $m \in \mathrm{W}_{n}(a, X, Z)$, then $f \in \mathrm{W}_{n}(a, X, Y)$.

Demonstraçãao. (i) Let $U \in \mathcal{T}_{f(a)}^{Y}$. Since $f \in \mathrm{C}_{m}(a, X, Y)$, then there exists $V \in \mathcal{T}_{m(a)}^{Z}$ such that $f\left(m^{-1}(V)\right) \subset U$ and, using Proposition 4 (iii), this is equivalent to $m^{-1}(V) \subset$ $f^{-1}(U)$. Similarly, since $m \in \mathrm{C}_{n}(a, X, Z)$, then there exists $W \in \mathcal{T}_{n(a)}^{R}$ such that $n^{-1}(W) \subset m^{-1}(V)$. Then $n^{-1}(W) \subset m^{-1}(V) \subset f^{-1}(U)$ and again using Proposition 4 (iii) we have that $f\left(n^{-1}(W)\right) \subset U$. Therefore $f \in \mathrm{C}_{n}(a, X, Y)$.

(ii) According to Theorem 4 (ii), if $m \in \mathrm{S}_{n}(a, X, Z)$, then $m \in \mathrm{C}_{n}(a, X, Z)$. Hence, using the hypotheses and the previous item (i), it is concluded that $f \in \mathrm{C}_{n}(a, X, Y)$ and consequently, using Theorem 4 (ii), $f \in \mathrm{W}_{n}(a, X, Y)$. In addition, $n$ is injective, since $n \in \mathbf{S}^{m}(a, X, Z)$. Therefore, $f \in \mathrm{S}_{n}(a, X, Y)$. 
(iii) Let $U \in \mathcal{T}_{f(a)}^{Y}$. Since $f \in \mathrm{C}_{m}(a, X, Y)$, then there exists $V \in \mathcal{T}_{m(a)}^{Z}$ satisfying the condition $m^{-1}(V) \subset f^{-1}(U)$. Since $m \in \mathrm{W}_{n}(a, X, Z)$, then there exists $W \in \mathcal{T}_{n(a)}^{R}$ such that $W \cap n(X) \subset n\left(m^{-1}(V)\right)$. Remember, from Proposition $4(\mathrm{i})$ is valid that $W \cap n(X)=$ $n\left(n^{-1}(W) \cap X\right)=n\left(n^{-1}(W)\right)$. Thus, $n\left(n^{-1}(W)\right) \subset n\left(m^{-1}(V)\right)$. It follows from Proposition 1 (ii) that if $m^{-1}(V) \subset f^{-1}(U)$, then $n\left(m^{-1}(V)\right) \subset n\left(f^{-1}(U)\right)$. Thus, $W \cap n(X)=n\left(n^{-1}(W)\right) \subset n\left(m^{-1}(V)\right) \subset n\left(f^{-1}(U)\right)$. Therefore, $f \in \mathrm{W}_{n}(a, X, Y)$.

Corollary 2. Let $X$ be a non-empty set and let $\left(Y, \mathcal{T}^{Y}\right),\left(Z, \mathcal{T}^{Z}\right)$ and $\left(R, \mathcal{T}^{R}\right)$ be topological spaces. Let $a \in X$ and let $f: X \rightarrow\left(Y, \mathcal{T}^{Y}\right), m: X \rightarrow\left(Z, \mathcal{T}^{Z}\right)$ and $n: X \rightarrow\left(R, \mathcal{T}^{R}\right)$ be functions. The following statements hold true:

(i) If $f \in \mathrm{S}_{m}(a, X, Y)$ and $m \in \mathrm{S}_{n}(a, X, Z)$, then $f \in \mathrm{S}_{n}(a, X, Y)$.

(ii) If $f \in \mathrm{S}_{m}(a, X, Y)$ and $m \in \mathrm{C}_{n}(a, X, Z)$, then $f \in \mathrm{C}_{n}(a, X, Y)$.

(iii) If $f \in \mathrm{S}_{m}(a, X, Y)$ and $m \in \mathrm{W}_{n}(a, X, Z)$, then $f \in \mathrm{W}_{n}(a, X, Y)$.

Demonstração. The statements follow from Theorem 7 combined with Theorem 4.

Example 17. Consider a set $X=\{a, b\}$, with $a \neq b$, equipped with the Sierpinski topology $\mathcal{S}=\{\emptyset,\{a\}, X\}$. Consider also the identity function $i d: X \rightarrow(X, \mathcal{S})$, the permutation function $p: X \rightarrow(X, \mathcal{S})$ and the function $c: X \rightarrow(X, \mathcal{S})$ given by $c(a)=c(b)=a$. Note that $\mathcal{S}_{a}=\{\{a\}, X\}$. From Proposition 14. we have that $i d \in \mathrm{W}_{c}(a, X, X)$. Moreover, we have $\{a\} \in \mathcal{S}_{a}$, but $i d(X)=X \not \subset\{a\}$. Hence, using contrapositive of item ii) of Proposition 13 it follows that id $\notin \mathrm{C}_{c}(a, X, X)$. Moreover, using Proposition $13(i)$, we have that $c \in \mathrm{C}_{p}(a, X, X)$ and, consequently, $c \in \mathrm{S}_{p}(a, X, X)$, since $p$ is injective. We know from Example 10 that $i d \notin \mathrm{W}_{p}(a, X, X)$. Therefore, $i d \in \mathrm{W}_{c}(a, X, X) \backslash \mathrm{C}_{c}(a, X, X)$ and $c \in \mathrm{S}_{p}(a, X, X)$, but $i d \notin \mathrm{W}_{p}(a, X, X)$.

The next result relates generalized continuity in the wide and strict sense with open functions and usual continuity, respectively. For this, note that considering $X$ a non-empty set, $\left(W, \mathcal{T}^{W}\right)$ a topological space and $h: X \rightarrow\left(W, \mathcal{T}^{W}\right)$ a function, then the family of subsets of $X$ given by

$$
\mathcal{T}^{h}=\left\{h^{-1}(U) \in \mathcal{P}(X): U \in \mathcal{T}^{W}\right\}
$$

is a topology in $X$, called topology induced by function $h$. Consider also $\mathcal{S}$ and $\mathcal{T}$ being topologies equipping the set $X$. The topology $\mathcal{S}$ is weaker (coarser or smaller) than the topology $\mathcal{T}$ if, and only if, $\mathcal{S} \subset \mathcal{T}$. 
Theorem 8. Let $\left(X, \mathcal{T}^{X}\right),\left(Y, \mathcal{T}^{Y}\right)$ and $\left(Z, \mathcal{T}^{Z}\right)$ be topological spaces and let $f: X \rightarrow$ $\left(Y, \mathcal{T}^{Y}\right)$ and $n: X \rightarrow\left(Z, \mathcal{T}^{Z}\right)$ be functions. The following statements hold true:

(i) If $f \in \mathrm{W}_{n}(X, Y)$ and $\mathcal{T}^{X} \subset \mathcal{T}^{f}$, then $n:\left(X, \mathcal{T}^{X}\right) \rightarrow\left(n(X), \mathcal{T}^{n(X)}\right)$ is open.

(ii) If $f \in \mathrm{S}_{n}(X, Y)$ and $\mathcal{T}^{n} \subset \mathcal{T}^{X}$, then $f:\left(X, \mathcal{T}^{X}\right) \rightarrow\left(Y, \mathcal{T}^{Y}\right)$ is continuous.

Demonstração. (i) Let $A \in \mathcal{T}^{X}$. By hypothesis, we have that $\mathcal{T}^{X} \subset \mathcal{T}^{f}$, then $A \in \mathcal{T}^{f}$ and therefore there exists $U \in \mathcal{T}^{Y}$ such that $A=f^{-1}(U)$. Since $f \in \mathrm{W}_{n}(X, Y)$, it follows from Theorem 6 that $n(A)=n\left(f^{-1}(U)\right) \in \mathcal{T}^{n(X)}$, that is, $n(A)$ is open subset of the image $n(X)$. Therefore, $n:\left(X, \mathcal{T}^{X}\right) \rightarrow\left(n(X), \mathcal{T}^{n(X)}\right)$ is an open function.

(ii) Let $U \in \mathcal{T}^{Y}$. Since $f \in \mathrm{S}_{n}(X, Y)$, then $n$ is injective and $f \in \mathrm{W}_{n}(X, Y)$. It follows from Theorem 6 that $n\left(f^{-1}(U)\right) \in \mathcal{T}^{n(X)}$, that is, there exists $W \in \mathcal{T}^{Z}$ such that $n\left(f^{-1}(U)\right)=W \cap n(X)$. Since $n$ is injective, using Proposition 3 (ii) and Proposition 2 (iii), we have that

$$
\begin{aligned}
f^{-1}(U) & =n^{-1}\left(n\left(f^{-1}(U)\right)=n^{-1}(W \cap n(X))\right. \\
& =n^{-1}(W) \cap n^{-1}(n(X))=n^{-1}(W) \cap X=n^{-1}(W) .
\end{aligned}
$$

Since $n^{-1}(W) \in \mathcal{T}^{n}$ and $\mathcal{T}^{n} \subset \mathcal{T}^{X}$, then $f^{-1}(U) \in \mathcal{T}^{X}$. Therefore, $f$ is continuous

\section{Continuity in the sense of Kupka}

The concept of similar functions was presented by Kupka in [5]. Subsequently, Kupka renames this concept for the term generalized continuity of a function with respect to another function (see [6], [7] and [8]). Now, the notion of similar functions or generalized continuity in the sense of Kupka will be presented and then a brief analysis will be made to point out aspects in which concept given by Kupka differs to the concept of generalized continuity proposed in this article. Some examples that show that the two concepts are not equivalent will also be presented.

Definition 16. Let $\left(X, \mathcal{T}^{X}\right),\left(Y, \mathcal{T}^{Y}\right)$ and $\left(Z, \mathcal{T}^{Z}\right)$ be topological spaces, let $a \in X$ and let $f:\left(X, \mathcal{T}^{X}\right) \rightarrow\left(Y, \mathcal{T}^{Y}\right)$ and $n:\left(X, \mathcal{T}^{X}\right) \rightarrow\left(Z, \mathcal{T}^{Z}\right)$ be functions. The function $f$ is said to be $n$-continuous at $a$ in the sense of Kupka if for every net $\left(x_{\lambda}\right)_{\lambda \in \Lambda}$ converging to a, the following holds:

If the net $\left(n\left(x_{\lambda}\right)\right)_{\lambda \in \Lambda}$ converges in $Z$, then the net $\left(f\left(x_{\lambda}\right)\right)_{\lambda \in \Lambda}$ converges in $Y$. 
Here we point out the first visible difference between the continuity in the sense of Kupka and the generalized continuity developed from the idea of generalized limit. The concept given by Kupka requires topological structure in the domain of functions continuator $n$ and continuant $f$. On the other hand, for the generalized continuity proposed in this article we do not need topological structure (or any other structure) in these domains.

The concept of generalized continuity proposed by this article can be characterized by nets, as proved in Theorem 5, and this allows a more accurate comparison between the two concepts of continuity mentioned. Note that for continuity in the sense of Kupka, it is required that the nets given in the domain of functions $n$ and $f$ have convergence to the point $a$ of reference, however it is only required that if there is convergence of the respective nets mapped in the codomain of the continuator $n$, then there should be convergence of the respective nets mapped in the codomain of the continuant $f$, without any determination for which points these nets should converge. In the concept of generalized continuity proposed here (via its characterization given by Theorem 5) there is no requirement of convergence of the nets given in the domain of $n$ and $f$ (since there is no topological structure in the domain), but there is the explicit requirement that if the respective nets mapped in the codomain of the continuator $n$ converge to $n(a)$, then the respective nets mapped in the codomain of the continuant $f$ should converge to $f(a)$.

Kupka also presented a second notion of continuity in [5], in which a topological structure is not required in the domain shared by continuator and continuant, but the condition (39) is still maintained (i.e., only free convergence is required for the nets mapped by continuator and continuant), unlike the notion of generalized continuity presented in this article, in which the mapped nets converge to specified points, namely, $n(a)$ and $f(a)$.

Definition 17. Let $X$ be a non-empty set, let $\left(Y, \mathcal{T}^{Y}\right)$ and $\left(Z, \mathcal{T}^{Z}\right)$ be topological spaces and let $f: X \rightarrow\left(Y, \mathcal{T}^{Y}\right)$ and $n: X \rightarrow\left(Z, \mathcal{T}^{Z}\right)$ be functions. The function $f$ is said to be $n$-constant on $X$ (in the sense of Kupka) if for every net $\left(x_{\lambda}\right)_{\lambda \in \Lambda}$ in $X$, the following holds:

If the net $\left(n\left(x_{\lambda}\right)\right)_{\lambda \in \Lambda}$ converges in $Z$, then the net $\left(f\left(x_{\lambda}\right)\right)_{\lambda \in \Lambda}$ converges in $Y$.

The notion given by Kupka in Definition 17 generates a subclass of functions of the class of the functions that satisfy the notion given in Definition 16 , because if $f$ is $n$ constant, then condition (39) is satisfied by a family of nets in $X$ that include the nets in $X$ that converge to $a$. Therefore, the function $f$ is also $n$-continuous at $a$ in the sense of Kupka.

Let $h:\left(X, \mathcal{T}^{X}\right) \rightarrow\left(Y, \mathcal{T}^{Y}\right)$ be a function and let $a \in X$. Denote the set of all nets in 
$X$ by $\mathfrak{N}(X)$ and consider the its following subsets:

$$
\begin{gathered}
\mathfrak{N}_{a}(X)=\left\{\left(x_{\lambda}\right)_{\lambda \in \Lambda} \in \mathfrak{N}(X): x_{\lambda} \rightarrow a\right\} \\
\mathfrak{N}^{h}(X)=\left\{\left(x_{\lambda}\right)_{\lambda \in \Lambda} \in \mathfrak{N}(X): h\left(x_{\lambda}\right) \text { is convergent }\right\}
\end{gathered}
$$

and

$$
\mathfrak{N}_{a}^{h}(X)=\left\{\left(x_{\lambda}\right)_{\lambda \in \Lambda} \in \mathfrak{N}(X): h\left(x_{\lambda}\right) \rightarrow h(a)\right\} .
$$

Note that $\mathfrak{N}_{a}^{h}(X)$ is a subset of $\mathfrak{N}^{h}(X)$.

Consider a continuant $f:\left(X, \mathcal{T}^{X}\right) \rightarrow\left(Y, \mathcal{T}^{Y}\right)$ and continuator $n:\left(X, \mathcal{T}^{X}\right) \rightarrow\left(Z, \mathcal{T}^{Z}\right)$. The set of all functions from $\left(X, \mathcal{T}^{X}\right)$ to $\left(Y, \mathcal{T}^{Y}\right)$ that are $n$-continuous at $a$ in the sense of Kupka will be denoted here by $\mathrm{K}_{n}(a, X, Y)$. Moreover, the set of all functions from $\left(X, \mathcal{T}^{X}\right)$ to $\left(Y, \mathcal{T}^{Y}\right)$ that are $n$-constant on $X$ will be denoted here by $\mathrm{cK}_{n}(X, Y)$. In other terms,

$$
\begin{gathered}
f \in \mathrm{cK}_{n}(X, Y) \Leftrightarrow \mathfrak{N}^{n}(X) \subset \mathfrak{N}^{f}(X), \\
f \in \mathrm{K}_{n}(a, X, Y) \Leftrightarrow \mathfrak{N}_{a}(X) \cap \mathfrak{N}^{n}(X) \subset \mathfrak{N}^{f}(X)
\end{gathered}
$$

and

$$
f \in \mathrm{C}_{n}(a, X, Y) \Leftrightarrow \mathfrak{N}_{a}^{n}(X) \subset \mathfrak{N}_{a}^{f}(X)
$$

Example 18. Consider a set $X=\{a, b\}$, with $a \neq b$, and consider the Sierpinski topology $\mathcal{S}=\{\emptyset,\{a\}, X\}$, the discrete topology $\mathcal{D}=\{\emptyset,\{a\},\{b\}, X\}$ and the trivial topology $\mathcal{T}=\{\emptyset, X\}$. Take $f:(X, \mathcal{S}) \rightarrow(X, \mathcal{D})$ given by $f(a)=a$ and $f(b)=b$, and take $n:(X, \mathcal{S}) \rightarrow(X, \mathcal{S})$ given by $n(a)=a$ and $n(b)=b$. We have that $\mathcal{D}_{f(a)}=$ $\{\{a\}, X\}=\mathcal{S}_{n(a)}$. Hence, given $U \in \mathcal{D}_{f(a)}$, take $V=U \in \mathcal{S}_{n(a)}$ and consequently $f\left(n^{-1}(V)\right) \subset U$. Thus, $f \in \mathrm{C}_{n}(a, X, X)$. Now, consider the alternated sequence $\left(x_{n}\right)_{n \in \mathbb{N}}=(a, b, a, b, a, b, \ldots)$ in $X$. We have that $x_{n} \rightarrow b$ (i.e. $\left.n\left(x_{n}\right) \rightarrow n(b)\right)$ with respect the topology $\mathcal{S}$, but $\left(x_{n}\right)_{n \in \mathbb{N}}=\left(f\left(x_{n}\right)\right)_{n \in \mathbb{N}}$ is not convergent with respect the topology $\mathcal{D}$. Since $\left(x_{n}\right)_{n \in \mathbb{N}} \in \mathfrak{N}^{n}(X)$ and $\left(x_{n}\right)_{n \in \mathbb{N}} \notin \mathfrak{N}^{f}(X)$, then, $f \notin \mathrm{cK}_{n}(X, X)$. Therefore, $\mathrm{C}_{n}(a, X, X) \not \subset \mathrm{cK}_{n}(X, X)$.

Example 19. Consider a set $X=\{a, b\}$, with $a \neq b$, and consider the following topologies: $\mathcal{S}=\{\emptyset,\{a\}, X\}, \mathcal{R}=\{\emptyset,\{b\}, X\}$ and $\mathcal{D}=\{\emptyset,\{a\},\{b\}, X\}$. Take $f:(X, \mathcal{R}) \rightarrow(X, \mathcal{D})$ given by $f(a)=a$ and $f(b)=b$, and take $n:(X, \mathcal{R}) \rightarrow(X, \mathcal{S})$ given by $n(a)=a$ and $n(b)=b$. As in the previous example, we have have that $\mathcal{D}_{f(a)}=\{\{a\}, X\}=\mathcal{S}_{n(a)}$ and therefore $f \in \mathrm{C}_{n}(a, X, X)$. Considering the alternated sequence $\left(x_{n}\right)_{n \in \mathbb{N}}=(a, b, a, b, a, b, \ldots)$ in $X$, we have that $x_{n} \rightarrow a$ with respect the topology $\mathcal{R}$ and $x_{n} \rightarrow b$ (i.e. $n\left(x_{n}\right) \rightarrow n(b)$ ) with respect the topology $\mathcal{S}$, but $\left(x_{n}\right)_{n \in \mathbb{N}}=\left(f\left(x_{n}\right)\right)_{n \in \mathbb{N}}$ is not convergent with respect the topology $\mathcal{D}$. Hence, since 
$\left(x_{n}\right)_{n \in \mathbb{N}} \in \mathfrak{N}_{a}(X) \cap \mathfrak{N}^{n}(X)$ and $\left(x_{n}\right)_{n \in \mathbb{N}} \notin \mathfrak{N}^{f}(X)$, then, $f \notin \mathrm{K}_{n}(a, X, X)$. Therefore, $\mathrm{C}_{n}(a, X, X) \not \subset \mathrm{K}_{n}(a, X, X)$.

Example 20. Consider a set $X=\{a, b\}$, with $a \neq b$, equipped with the Sierpinski topology $\mathcal{S}=\{\emptyset,\{a\}, X\}$. Consider also the identity function id: $(X, \mathcal{S}) \rightarrow(X, \mathcal{S})$ and the permutation function $p:(X, \mathcal{S}) \rightarrow(X, \mathcal{S})$ given by $p(a)=b$ and $p(b)=a$. Let $\left(x_{\lambda}\right)_{\lambda \in \Lambda} \in \mathfrak{N}_{a}(X)$. Since $U=X$ is unique in $\mathcal{S}_{b}$, then $p\left(x_{\lambda}\right) \in U$, for all $\lambda \in \Lambda$. Therefore, $p\left(x_{\lambda}\right) \rightarrow b$ with respect to the topology $\mathcal{S}$ and $\left(x_{\lambda}\right)_{\lambda \in \Lambda} \in \mathfrak{N}^{p}(X)$. Of course, $x_{\lambda} \rightarrow$ a with respect to the topology $\mathcal{S}$, then $\left(x_{\lambda}\right)_{\lambda \in \Lambda} \in \mathfrak{N}^{i d}(X)$. Thus, $\mathfrak{N}_{a}(X) \cap \mathfrak{N}^{p}(X) \subset$ $\mathfrak{N}^{i d}(X)$, that is, id $\in \mathrm{K}_{p}(a, X, X)$. We have already observed in Example 10 that id $\notin$ $\mathrm{C}_{p}(a, X, X)$. Therefore, $\mathrm{K}_{p}(a, X, X) \not \subset \mathrm{C}_{p}(a, X, X)$.

Example 21. It is immediate to verify that any continuous function $f:\left(X, \mathcal{T}^{X}\right) \rightarrow$ $\left(Y, \mathcal{T}^{Y}\right)$ satisfies $f \in \mathrm{C}_{i d}(a, X, Y) \cap \mathrm{K}_{i d}(a, X, Y)$.

The previous examples show that the concept of generalized continuity proposed in this text is not a proper subclass of the concept of continuity in the sense of Kupka and the latter also is not a proper subclass of the first. Moreover, these concepts are not equivalent, although they intersect.

The following proposition establishes some conditions in which there is some relationship between the concept of continuity given by Kupka and the concept of generalized continuity proposed here.

Proposition 15. Let $\left(X, \mathcal{T}^{X}\right),\left(Y, \mathcal{T}^{Y}\right)$ and $\left(Z, \mathcal{T}^{Z}\right)$ be topological spaces and consider $n:\left(X, \mathcal{T}^{X}\right) \rightarrow\left(Z, \mathcal{T}^{Z}\right)$ a function. The following statements hold true:

(i) $\mathrm{cK}_{n}(X, Y) \subset \bigcup_{a \in X} \mathrm{~K}_{n}(a, X, Y)$.

(ii) If $f \in \mathrm{cK}_{n}(X, Y)$ and $\mathfrak{N}^{f}(X)=\mathfrak{N}_{a}^{f}(X)$, then $f \in \mathrm{C}_{n}(a, X, Y)$.

(iii) If $f \in \mathrm{C}_{n}(a, X, Y)$ and $\mathfrak{N}^{n}(X)=\mathfrak{N}_{a}^{n}(X)$, then $f \in \mathrm{cK}_{n}(X, Y)$.

(iv) If $\mathfrak{N}^{n}(X)=\mathfrak{N}_{a}^{n}(X)$ and $\left(Z, \mathcal{T}^{Z}\right)$ is Hausdorff space, then $n$ is a constant function.

Demonstração. (i) Let $a \in X$. If $f \in \mathrm{cK}_{n}(X, Y)$, then $\mathfrak{N}^{n}(X) \subset \mathfrak{N}^{f}(X)$. Consequently $\mathfrak{N}_{a}(X) \cap \mathfrak{N}^{n}(X) \subset \mathfrak{N}^{n}(X) \subset \mathfrak{N}^{f}(X)$ and hence $f \in \mathrm{K}_{n}(a, X, Y)$. Thus, $\mathrm{cK}_{n}(X, Y) \subset \mathrm{K}_{n}(a, X, Y)$, for each $a \in X$. Therefore, $\mathrm{cK}_{n}(X, Y) \subset \bigcup_{a \in X} \mathrm{~K}_{n}(a, X, Y)$.

(ii) If $f \in \mathrm{cK}_{n}(X, Y)$, then $\mathfrak{N}^{n}(X) \subset \mathfrak{N}^{f}(X)$. It is immediate that $\mathfrak{N}_{a}^{n}(X) \subset \mathfrak{N}^{n}(X)$ and since by hypothesis $\mathfrak{N}^{f}(X)=\mathfrak{N}_{a}^{f}(X)$, it follows that $\mathfrak{N}_{a}^{n}(X) \subset \mathfrak{N}_{a}^{f}(X)$. Therefore, $f \in \mathrm{C}_{n}(a, X, Y)$. 
(iii) If $f \in \mathrm{C}_{n}(a, X, Y)$, then $\mathfrak{N}_{a}^{n}(X) \subset \mathfrak{N}_{a}^{f}(X)$. Since by hypothesis $\mathfrak{N}^{n}(X)=\mathfrak{N}_{a}^{n}(X)$ and as $\mathfrak{N}_{a}^{f}(X) \subset \mathfrak{N}^{f}(X)$, it follows that $\mathfrak{N}^{n}(X) \subset \mathfrak{N}^{f}(X)$. Therefore, $f \in \mathrm{cK}_{n}(X, Y)$.

(iv) Let $b \in X$ such that $b \neq a$. Taking the constant sequence $\left(x_{k}\right)_{k \in \mathbb{N}}=(b, b, b, b, b \ldots)$, it follows that $\left(n\left(x_{k}\right)\right)_{k \in \mathbb{N}}=(n(b), n(b), n(b), n(b), n(b) \ldots)$ is such that $n\left(x_{k}\right) \rightarrow n(b)$ and $\left(x_{k}\right)_{k \in \mathbb{N}} \in \mathfrak{N}^{n}(X)$. Hence, using hypothesis, we have that $\left(x_{k}\right)_{k \in \mathbb{N}} \in \mathfrak{N}_{a}^{n}(X)$ and therefore $n\left(x_{k}\right) \rightarrow n(a)$. Since $Z$ is Hausdorff space, then the convergent sequence $\left(x_{k}\right)_{k \in \mathbb{N}}$ has a single limit, that is, $n(a)=n(b)$. Therefore, $n(a)=n(b)$, for all $b \in X$, that is, $n$ is a constant function.

\section{Topological invariants and generalized continuity}

In this section it is verified that compactness and connectedness are adequately preserved through generalized continuity.

Let $X$ be a set. A collection $\mathcal{C}$ is said to be a cover of set $X$ if

$$
X \subset \bigcup_{U \in \mathcal{C}} U
$$

A collection $\mathcal{D}$ is said to be a subcover of a cover $\mathcal{C}$ of $X$ if $\mathcal{D} \subset \mathcal{C}$ and $\mathcal{D}$ is a cover of $X$.

Definition 18. Let $\left(X, \mathcal{T}^{X}\right)$ be a topological space and let $K$ be a subset of $X$. A collection $\mathcal{C}$ is said to be a open cover of set $K$ with respect to $\mathcal{T}^{X}$ if $\mathcal{C}$ is a cover of $K$ and $\mathcal{C} \subset \mathcal{T}^{X}$.

Definition 19. Let $\left(X, \mathcal{T}^{X}\right)$ be a topological space and let $K$ be a subset of $X$. The set $K$ is said to be compact with respect to $\mathcal{T}^{X}$ if each open cover $\mathcal{C}$ of $K$ with respect to $\mathcal{T}^{X}$ has a finite subcover.

In other words, a subset $K$ of a topological space $\left(X, \mathcal{T}^{X}\right)$ is called compact if for every open cover $\mathcal{C}$ of $K$ with respect to $\mathcal{T}^{X}$, there exists a finite subset $\mathcal{F}$ of $\mathcal{C}$ such that

$$
K \subset \bigcup_{U \in \mathcal{F}} U
$$

Theorem 9. Let $X$ be a non-empty set, let $\left(Y, \mathcal{T}^{Y}\right)$ and $\left(Z, \mathcal{T}^{Z}\right)$ be topological spaces, let $f: X \rightarrow\left(Y, \mathcal{T}^{Y}\right)$ and $n: X \rightarrow\left(Z, \mathcal{T}^{Z}\right)$ be functions. If $f$ é $n$-continuous and $n(X)$ is compact with respect to $\mathcal{T}^{Z}$, then $f(X)$ is compact with respect to $\mathcal{T}^{Y}$. 
Demonstração. Let $\mathcal{C}$ be a open cover of $f(X)$ with respect to $\mathcal{T}^{Y}$. By hypothesis $f \in \mathrm{C}_{n}(X, Y)$, then $f \in \mathrm{C}_{n}(a, X, Y)$, for all $a \in X$. Since $f(X) \subset \bigcup_{U \in \mathcal{C}} U$ and $\mathcal{C} \subset \mathcal{T}^{Y}$, then for each $a \in X$, there exists $U_{a} \in \mathcal{C}$ such that $f(a) \in U_{a}$ and since $f \in \mathrm{C}_{n}(a, X, Y)$, it follows that there exists $V_{a} \in \mathcal{T}_{n(a)}^{Z}$ such that $f\left(n^{-1}\left(V_{a}\right)\right) \subset U_{a}$. Consider $\mathcal{D}$ being the collection of all open sets $V_{a}$ indexed by $a \in X$ and previously described. It is easy to see that $n(X) \subset \bigcup_{a \in X} V_{a}$ and therefore the collection $\mathcal{D}$ is a open cover of $n(X)$ with respect to $\mathcal{T}^{Z}$. Since $n(X)$ is compact with respect to $\mathcal{T}^{Z}$, then the open cover $\mathcal{D}$ of $n(X)$ has a finite subcover, that is, there is a finite amount of points $a_{1}, \ldots, a_{k} \in X$ such that $n(X) \subset \bigcup_{i=1}^{k} V_{a_{i}}$. It follows from Proposition 3 (iii) and Proposition 2 (i) and (ii) that

$$
X=n^{-1}(n(X)) \subset n^{-1}\left(\bigcup_{i=1}^{k} V_{a_{i}}\right)=\bigcup_{i=1}^{k} n^{-1}\left(V_{a_{i}}\right)
$$

and this combined with Proposition 1 (ii) and (iii) gives us

$$
f(X) \subset f\left(\bigcup_{i=1}^{k} n^{-1}\left(V_{a_{i}}\right)\right)=\bigcup_{i=1}^{k} f\left(n^{-1}\left(V_{a_{i}}\right)\right) \subset \bigcup_{i=1}^{k} U_{a_{i}}
$$

Thus, since $U_{a_{1}}, \ldots, U_{a_{k}} \in \mathcal{C}$ and $f(X) \subset \bigcup_{i=1}^{k} U_{a_{i}}$, then $\mathcal{C}$ has a finite subcover. Therefore, $f(X)$ is compact with respect to $\mathcal{T}^{Y}$.

The conclusion of Theorem 9 does not remain valid if we replace the hypothesis that the continuant $f$ is $n$-continuous by the hypothesis of it to be widely $n$-continuous. In fact, considering as continuant the identity function $i d: \mathbb{R} \rightarrow \mathbb{R}$, with $\mathbb{R}$ equipped with the usual topology, and considering as continuator a constant function $n(x)=c$, for all $x \in \mathbb{R}$, we have by Proposition 14 that $i d \in \mathrm{W}_{n}(X, Y)$. In addition $n(\mathbb{R})=\{c\}$ is compact, because it is a finite set, but $i d(\mathbb{R})=\mathbb{R}$ is not compact, because it is not bounded set.

Theorem 10. Let $X$ be a non-empty set, let $\mathbb{R}$ be equipped with usual topology, let $\left(Z, \mathcal{T}^{Z}\right)$ be a topological space, let $f: X \rightarrow \mathbb{R}$ and $n: X \rightarrow\left(Z, \mathcal{T}^{Z}\right)$ be functions. If $f$ is n-continuous and $n(X)$ is compact with respect to $\mathcal{T}^{Z}$, then $f$ assumes maximum value and minimum value.

Demonstração. It follows from Theorem 9 that $f(X)$ is compact. Therefore, $f(X)$ is bounded subset of $\mathbb{R}$ and consequently such subset admits supremum and infimum, denoted respectively by $\sup f$ and $\inf f$. For each $k \in \mathbb{N}$, there exists a $x_{k} \in X$ such that $\sup f-\frac{1}{k}<f\left(x_{k}\right) \leq \sup f$. Thus, there exists a sequence $\left(x_{k}\right)_{k \in \mathbb{N}}$ in $X$ such 
as $0 \leq \sup f-f\left(x_{k}\right)<\frac{1}{k}$. Because $n(X)$ is compact, it follows that the sequence (net) $\left(n\left(x_{k}\right)\right)_{k \in \mathbb{N}}$ admits a subsequence (subnet) $\left(n\left(x_{k_{i}}\right)\right)_{i \in \mathbb{N}}$, which is convergent to some $z \in n(X)$. Since $z \in n(X)$, then there exists $a \in X$ such that $n(a)=z$. Then, $n\left(x_{k_{i}}\right) \rightarrow n(a)$ and as $f$ is $n$-continuous, follows from Theorem 5 that $f\left(x_{k_{i}}\right) \rightarrow f(a)$. On the other hand, since $0 \leq \sup f-f\left(x_{k_{i}}\right)<\frac{1}{k_{i}}$ and $\frac{1}{k_{i}} \rightarrow 0$, we have that $f\left(x_{k_{i}}\right) \rightarrow \sup f$. It follows from the uniqueness of limit in $\mathbb{R}$ that $f(a)=\sup f$, that is, $\sup f \in f(X)$ and therefore $f$ assumes maximum value. Similarly, it is concluded that $f$ assumes minimum value.

Definition 20. Let $\left(X, \mathcal{T}^{X}\right)$ be a topological space and let $C$ be a subset of $X$.

- The set $X$ is said to be disconnected with respect to $\mathcal{T}^{X}$, if there exist $A, B \in \mathcal{T}^{X}$ such that they are disjoint non-empty sets and $A \cup B=X$.

- The set $X$ is said to be connected with respect to $\mathcal{T}^{X}$, if it is not disconnected with respect to $\mathcal{T}^{X}$.

- The subset $C$ of $X$ is said to be connected with respect to $\mathcal{T}^{X}$ if it as topological subspace of $\left(X, \mathcal{T}^{X}\right)$ is connected with respect to induced topology by $\mathcal{T}^{X}$.

Theorem 11. Let $X$ be a non-empty set, let $\left(Y, \mathcal{T}^{Y}\right)$ and $\left(Z, \mathcal{T}^{Z}\right)$ be topological spaces, let $f: X \rightarrow\left(Y, \mathcal{T}^{Y}\right)$ and $n: X \rightarrow\left(Z, \mathcal{T}^{Z}\right)$ be functions. If $f$ is $n$-continuous and $n(X)$ is connected with respect to $\mathcal{T}^{Z}$, then $f(X)$ is connected with respect to $\mathcal{T}^{Y}$.

Demonstração. Let $A, B \in \mathcal{T}^{f(X)}$ such that $A \cap B=\emptyset, A \cup B=f(X)$ and $A \neq \emptyset$. Since by hypothesis $f$ is $n$-continuous, then for each $a \in f^{-1}(A)$, there exists a $V_{a} \in \mathcal{T}_{n(a)}^{Z}$ such that $f\left(n^{-1}\left(V_{a}\right)\right) \subset A$, which is equivalent to $n^{-1}\left(V_{a}\right) \subset f^{-1}(A)$. Similarly, for each $b \in f^{-1}(B)$, there exists a $W_{b} \in \mathcal{T}_{n(b)}^{Z}$ such that $n^{-1}\left(W_{b}\right) \subset f^{-1}(B)$. Consider the sets

$$
V=\left(\bigcup_{a \in f^{-1}(A)} V_{a}\right) \cap n(X) \quad \text { and } \quad W=\left(\bigcup_{b \in f^{-1}(B)} W_{b}\right) \cap n(X)
$$

We have that $V \in \mathcal{T}^{n(X)}$ and $W \in \mathcal{T}^{n(X)}$. It follows from Proposition 2 (ii) and (iii) and Proposition 3 (iii) that

$$
\begin{aligned}
n^{-1}(V) & =n^{-1}\left(\left(\bigcup_{a \in f^{-1}(A)} V_{a}\right) \cap n(X)\right)=n^{-1}\left(\bigcup_{a \in f^{-1}(A)} V_{a}\right) \cap n^{-1}(n(X)) \\
& =\left(\bigcup_{a \in f^{-1}(A)} n^{-1}\left(V_{a}\right)\right) \cap X=\bigcup_{a \in f^{-1}(A)} n^{-1}\left(V_{a}\right) \subset f^{-1}(A)
\end{aligned}
$$


and given any $x \in f^{-1}(A)$, then there exists a $V_{x} \in \mathcal{T}_{n(x)}^{Z}$ such that $n^{-1}\left(V_{x}\right) \subset f^{-1}(A)$ and consequently $n(x) \in V_{x} \cap n(X) \subset V$ and $x \in n^{-1}(V)$, that is, $f^{-1}(A) \subset n^{-1}(V)$. Therefore $n^{-1}(V)=f^{-1}(A)$ and, similarly, $n^{-1}(W)=f^{-1}(B)$. Thus, it follows from Proposition 2(iii), Proposition 1(iv) and Proposition 3 (iv) that

$$
\begin{aligned}
f\left(n^{-1}(V \cap W)\right) & =f\left(n^{-1}(V) \cap n^{-1}(W)\right) \subset f\left(n^{-1}(V)\right) \cap f\left(n^{-1}(W)\right) \\
& =f\left(f^{-1}(A)\right) \cap f\left(f^{-1}(B)\right) \subset A \cap B=\emptyset,
\end{aligned}
$$

that is, $f\left(n^{-1}(V \cap W)\right)=\emptyset$, which is equivalent by Proposition 1 (i) to $n^{-1}(V \cap W)=\emptyset$ and this in turn is equivalent to $n\left(n^{-1}(V \cap W)\right)=\emptyset$. Hence, using Proposition 4 (i) we have that

$$
V \cap W=(V \cap W) \cap n(X)=n\left(n^{-1}(V \cap W) \cap X\right)=n\left(n^{-1}(V \cap W)\right)=\emptyset .
$$

Using Proposition 3 (iii) and Proposition 2(iii), we have that

$$
\begin{aligned}
X & \left.=f^{-1}(f(X))=f^{-1}(A \cup B)\right)=f^{-1}(A) \cup f^{-1}(B) \\
& =n^{-1}(V) \cup n^{-1}(W)=n^{-1}(V \cup W),
\end{aligned}
$$

and from Proposition 3 (iv) it implies that

$$
n(X)=n\left(n^{-1}(V \cup W)\right) \subset V \cup W \subset n(X),
$$

that is, $V \cup W=n(X)$. In addition, if $A \neq \emptyset$ and $A \subset f(X)$, then $n^{-1}(V)=f^{-1}(A) \neq \emptyset$ and $n\left(n^{-1}(V)\right) \neq \emptyset$. Since $n\left(n^{-1}(V)\right) \subset V$ and $n\left(n^{-1}(V)\right) \neq \emptyset$, then $V \neq \emptyset$. Thus, if $V, W \in \mathcal{T}^{n(X)}, V \cap W=\emptyset, V \cup W=n(X), V \neq \emptyset$ and by hypothesis $n(X)$ is connected, then $W=\emptyset$. Hence, $f^{-1}(B)=n^{-1}(W)=\emptyset$ and since $B \subset f(X)$, then $B=\emptyset$ (otherwise, we would have to $f^{-1}(B) \neq \emptyset$ ). Thus, given $A, B \in \mathcal{T}^{f(X)}$ with $A \cap B=\emptyset, A \cup B=f(X)$ and $A \neq \emptyset$, it is concluded that $B=\emptyset$. Therefore, $f(X)$ is connected.

The conclusion of Theorem 11 does not remain valid if we replace the hypothesis that the continuant $f$ is $n$-continuous by the hypothesis of it to be widely $n$-continuous. In fact, considering $\mathbb{R}$ equipped with the usual topology and considering as continuant $f: \mathbb{R}-\{0\} \rightarrow \mathbb{R}$ given by $f(x)=x$ and as continuator $n: \mathbb{R}-\{0\} \rightarrow \mathbb{R}$ given by $n(x)=|x|$, it is similar to Example 8 that $f \in \mathrm{W}_{n}(X, Y)$ and in addition $n(\mathbb{R} \backslash\{0\})=$ $(0, \infty)$ is connected, but $f(\mathbb{R} \backslash\{0\})=(-\infty, 0) \cup(0, \infty)$ is disconnected. 


\section{Conclusion}

It is concluded by this work that it is possible to establish a definition of generalized continuity through generalized limits and that this definition leads to some results similar to those resulting from usual continuity, especially results involving compactness and connectedness.

It should be noted that generalized continuity and usual continuity are not equivalent concepts. It can be said that generalized continuity is more flexible than usual continuity, in the sense that discontinuous functions (in the usual sense) can be $n$-continuous, depending on the function $n$ considered.

Another aspect, which is no less important, is that unlike the usual continuity, the generalized continuity presented in this article does not require topological structure in the domains of functions, only in their codomains. Thus, it is possible to have an $n$ continuous function assuming maximum value and minimum value, regardless of whether the domain is compact or has topological structure, it is sufficient to have compactness in the image of the function $n$ considered.

\section{References}

[1] BOtelho, G. M. A.; PELleGRinO, D. M.; TEIXEIRA, E. V. Fundamentos de Análise Funcional, 2. ed. Rio de Janeiro: Ed. SBM, 2015.

[2] BRAZ, J. H. S.; VIEIRA, M. G. O. Limites generalizados de funções, In: V SEMAP, n. 5, 2014. Anais da V SEMAP. Available in: http://www . semap. facip. ufu.br/node/30, Accessed on 11/19/2020.

[3] COLONIUS, F.; KIZIL E.; SAN MARTIN L. A. B. Covering space for monotonic homotopy of trajectories of control system. J. Differential Equations, v. 216, n. 2 , p. 324-353, 2005.

[4] KÜHLKAMP, N. Introdução à Topologia Geral, 2. ed. Florianópolis: Ed. da UFSC, 2002.

[5] KUPKA, I. On similarity of functions, Top. Proc., v. 36, p. 173-187, 2010.

[6] KUPKA, I. Similar functions and their properties, Tatra Mt. Math. Publ., v. 55, p. 47-56, 2013.

[7] KUPKA, I. Measurability of similar functions, Ann. Acad. Sci. Fenn. Math. Diss., v. 42, p. 803-808, 2017. 
[8] KUPKA, I. Generalized derivative and generalized continuity, Tatra Mt. Math. Publ., v. 74, p. 77-84, 2019.

[9] MUNKRES, J. R. Topology, 2. ed. London: Pearson, 2013.

[10] VIEIRA, M. G. O.; KIZIL, E; CATUOGNO, P. J. Monotonic homotopy for trajectories of Young systems. J. Dyn. Control Syst., v. 19, n. 3, p. 405-420, 2013.

[11] VIEIRA, M. G. O.; KIZIL, E; CATUOGNO, P. J. Regular trajectories of Young systems. J. Dyn. Control Syst., v. 21, n. 1, p. 1-21, 2015.

[12] VIEIRA, M. G. O. Continuidade no contexto de limites generalizados, In: VII SEMAP, n. 7, 2016. Anais da VII SEMAP. Available in: http: / / www . semap. facip.ufu.br/node/69. Accessed on 11/19/2020.

[13] WILLARD, S. General Topology, Reading, Massachusetts: Addison- Wesley Publishing Company, 1970.

口

Submitted on June 7, 2020.

Accepted on November 19, 2020. 